INRA Prod. Anim., 2011, 24 (5), 433-446

\title{
Influences des régimes et de leur fermentation dans le rumen sur la production de méthane par les ruminants
}

\author{
D. SAUVANT ${ }^{1,2}$, S. GIGER-REVERDIN ${ }^{1,2}$, A. SERMENT ${ }^{1,2}$, L. BROUDISCOU ${ }^{1,2}$
}

1 INRA, UMR791 Modélisation Systémique Appliquée aux Ruminants, 16 rue Claude Bernard, F-75005 Paris, France

2 AgroParis Tech, Modélisation Systémique Appliquée aux Ruminants, 16 rue Claude Bernard, F-75005 Paris, France

Courriel :daniel.sauvant@agroparistech.fr

En relation avec les préoccupations environnementales, la compréhension des déterminants de la production de méthane dans le rumen est devenue un sujet majeur de la nutrition des ruminants. Cet article présente les phénomènes digestifs et fermentaires conduisant à la production de méthane. Il quantifie l'influence des principaux facteurs alimentaires sur ces fermentations et la production de méthane associée.

Les causes de variations de la production de méthane $\left(\mathrm{CH}_{4}\right)$ par les ruminants ont été considérées depuis des décennies en raison de la perte énergétique associée. Depuis 15-20 ans, en relation avec les préoccupations environnementales, il y a un net regain d'intérêt pour les recherches sur le $\mathrm{CH}_{4}$, et une envolée du nombre de publications sur la nutrition des ruminants comprenant le méthane parmi les mots-clés. En effet, une exploration par le «Web of Science» montre que le nombre d'articles publiés avec les mots-clés «méthane» et «ruminants» s'accroît exponentiellement, de près de $15 \%$ par an, depuis 1990. En outre, un récent congrès international a été consacré à cette question (numéro spécial d'Animal Feed Science and Technology, paru en 2011). Même si à une échelle globale, le $\mathrm{CH}_{4}$ issu des fermentations entériques des ruminants ne représente qu'une très faible contribution à l'effet de serre (moins de 1\%), ou aux Gaz à Effet de Serre (GES) (2 à 4\%), à l'échelle de l'exploitation bovine, il peut représenter plus de la moitié de la production des GES (Dollé et al 2009).

L'objectif de cet article est de faire le point sur les mécanismes et processus fermentaires majeurs dans le rumen liés à la production de $\mathrm{CH}_{4}$ et de mettre en évidence ses principaux contrôles alimentaires, de manière à pouvoir aboutir à des prévisions fiables. Cette approche nécessite de revisiter les fermentations ruminales et leurs relations avec la méthanogenèse, en privilégiant la valo- risation des résultats expérimentaux récents et l'interprétation de larges bases de données de manière à pouvoir disposer de relations génériques. Des relations essentielles de prévision du $\mathrm{CH}_{4}$ produit, utilisables en pratique, sont proposées dans cette étude.

\section{1 / Les phénomènes digestifs et fermentaires, et les contrô- les stochiométriques et ther- modynamiques liés au $\mathrm{CH}_{4}$}

\section{1 / Les méthodes d'études}

Ce travail a été conduit en s'appuyant sur des résultats d'études conduites in vitro ou in vivo. Les études in vitro consistent à faire incuber un substrat (ration, aliment, avec ou sans additif...) pendant une durée définie en présence d'un inoculum constitué de contenu ruminal dilué dans une solution tamponnée. La mesure du $\mathrm{CH}_{4}$ produit en termes d'intensité de production et de dynamique est possible par l'échantillonnage et l'analyse des gaz fermentaires produits au cours de l'incubation. Les études in vitro permettent de tester différents types de régimes rapidement. En outre, certaines mesures de bilans fermentaires (bilans C, H, N, Acides Gras Volatils (AGV) produits) ne peuvent être réalisées précisément que lors d'études conduites in vitro.

Néanmoins, il est nécessaire de vérifier que la production in vitro de $\mathrm{CH}_{4}$ est cohérente avec une production in vivo. Les études conduites in vivo avec mesure de la production de $\mathrm{CH}_{4}$ ont longtemps concerné uniquement des animaux placés en cages à bilan calorimétrique. La base «Rumener», développée depuis plus de 10 ans au sein de l'UMR INRA-AgroParisTech MoSAR, rassemble des résultats de mesures de bilans calorimétriques sur des ruminants (bovins, ovins et caprins; 167 publications et 1131 traitements correspondant principalement à des variations dans le régime alimentaire). Ces données permettent de disposer de résultats de production de $\mathrm{CH}_{4}$ et de l'ensemble des autres paramètres des bilans d'énergie ainsi que, pour les études récentes, des fermentations ruminales. Pour préciser certains aspects (influence de l'alimentation sur le profil des AGV du rumen...) et valider certains résultats de la base «Rumener», la base «Bovidig» de l'unité a également été mobilisée (534 publications et 2016 traitements). Cette dernière concerne des études de digestion effectuées sur des bovins recevant des régimes très variés. Pour ces deux bases, des codages spécifiques permettent d'interpréter les résultats sous différents angles. Le traitement statistique est conduit par métaanalyse compte tenu de l'hétérogénéité des données traitées et de manière à pouvoir dégager, en intra-expérience, des lois de réponse aux facteurs considérés, à valeur générique (Sauvant et al 2008). 


\section{2 / Les principales voies de dégradation des substrats de polymères glucidiques}

Les principales voies fermentaires du rumen sont bien connues, elles sont détaillées dans l'article de Popova et al (2011) de ce même numéro. Les polymères glucidiques (cellulose, hémicelluloses, amidon...) sont dégradés en molécules simples, les oligosides, puis en oses à 6 ou 5 atomes de carbone $(\mathrm{C})$. On parlera ensuite essentiellement de glucose. Ces oses sont ensuite métabolisés au sein des cellules microbiennes par le processus de la glycolyse qui aboutit à des molécules à $3 \mathrm{C}$, dont l'acide pyruvique qui constitue un «carrefour métabolique». En effet, à partir de l'acide pyruvique, plusieurs voies fermentaires peuvent se dérouler dans le rumen, conduisant principalement à la formation d'Acides Gras Volatils (AGV) : acétique (en $\mathrm{C}_{2}$ ), propionique (en $\mathrm{C}_{3}$ ) et butyrique (en $\mathrm{C}_{4}$ ). D'autres $\mathrm{AGV}$, dits mineurs, sont synthétisés. Ils possèdent 4 (iso-butyrique), 5 (valérique, iso-valérique) ou 6 (caproïque) atomes de $\mathrm{C}$. Ces AGV mineurs sont souvent regroupés en une seule molécule «moyenne» (AGV min) considérée à 5 , ou 5,5 atomes de $\mathrm{C}$ en moyenne. Enfin, du lactate est aussi formé et accumulé en très faible quantité et de façon transitoire après les repas, mis à part dans le cas de situations d'acidose aiguë (Sauvant et Peyraud 2010).

Ces différentes voies de dégradation et de fermentation sont associées à la synthèse et/ou à utilisation de transporteurs d'hydrogène moléculaire (désigné par $\mathrm{H}_{2}$ lié à son transporteur), d'énergie sous forme d'ATP et à des pertes de carbone $(\mathrm{C})$. Les principales voies fermentaires du glucose sont résumées dans le tableau 1. Pour chaque voie, il s'agit des valeurs les plus communément admises, certaines peuvent varier. Ainsi, selon les auteurs et la voie utilisée, la production d'ATP lorsque du glucose est fermenté en propionate est de 3 ou 4 selon qu'il est synthétisé par la voie du lactate ou par celle du succinate. De même, elle peut être de 1 ou 0
ATP par AGV mineur. Ces variantes soulèvent la question de la précision des interprétations énergétiques des fermentations du rumen. Toutes ces relations stœchiométriques ont été décrites de façon plus détaillées (Offner et Sauvant 2006)

La production de méthane fait partie des fermentations ruminales, le méthane est synthétisé par des microorganismes méthanogènes, les Archaea, selon la réaction suivante qui est forte consommatrice d' $\mathrm{H}_{2}$ :

$$
\mathrm{CO}_{2}+8 \mathrm{H} \rightarrow \mathrm{CH}_{4}+2 \mathrm{H}_{2} \mathrm{O}+1 \mathrm{ATP}
$$

Cependant, l'hydrogène peut également être recyclé par une voie non productrice de $\mathrm{CH}_{4}$, mais productrice d'acétate, l'acétogénèse, avec comme équation résultante :

\section{$2 \mathrm{CO}_{2}+8 \mathrm{H} \rightarrow$ acétate $+2 \mathrm{H}_{2} \mathrm{O}+0,25$ ATP}

Cette dernière voie est intéressante, car elle évite un gâchis de $\mathrm{C}$ et ne produit pas de $\mathrm{CH}_{4}$. Elle se rencontre principalement dans le gros intestin et caecum des tubes digestifs des herbivores et des omnivores.

\section{3 / Les principales combinai- sons entre voies fermentaires}

Ces différentes voies fermentaires se déroulent simultanément, et dans des proportions variables, en fonction de différents facteurs qui sont très liés à des pratiques alimentaires. Ces variations influencent la production de $\mathrm{CH}_{4}$. Les combinaisons les plus fréquentes de ces voies ont été proposées depuis longtemps (Wolin 1960) dans le but d'évaluer les bilans fermentaires moyens du rumen ou bien les rôles métaboliques de certains groupes dominants de microorganismes. La proposition la plus connue et appliquée a été celle de Murphy et al (1982), qui ont proposé de prévoir la stœchiométrie des AGV produits essentiellement en fonction des substrats fermentés (cellulose, hémicelluloses, amidon...) et des caractéristiques globales du régime alimentaire (riche ou pauvre

Tableau 1. Principales équations stœchiométriques simplifiées de la fermentation $d u$ glucose dans le rumen.

\begin{tabular}{l}
\hline Glucose $\rightarrow 2$ pyruvate $+2 \mathrm{H}_{2}+2 \mathrm{ATP}+2 \mathrm{H}_{2} \mathrm{O}$ \\
\hline Glucose $\rightarrow 2$ acétate $+4 \mathrm{H}_{2}+2 \mathrm{CO}_{2}-2 \mathrm{H}_{2} \mathrm{O}+4$ ATP \\
\hline Glucose $\rightarrow 2$ propionate $-2 \mathrm{H}_{2}+2 \mathrm{H}_{2} \mathrm{O}+4$ ATP \\
\hline Glucose $\rightarrow$ butyrate $+2 \mathrm{H}_{2}+2 \mathrm{CO}_{2}+3$ ATP \\
\hline Glucose $\rightarrow$ AGVmin $-\mathrm{H}_{2}+1 \mathrm{CO}_{2}+1$ ATP \\
\hline
\end{tabular}

en fourrages). D'autres relations assez comparables ont été proposées (Bannink et al 2008). Les limites de ces approches viennent principalement du fait que les substrats, considérés comme digérés dans le rumen, ne sont en général pas mesurés spécifiquement, ils sont donc estimés avec une large incertitude (exemple : les hémicelluloses...) et ne figurent pas dans les tables d'alimentation. Pour contourner cette difficulté, Nozière et al (2010) ont proposé une approche simple, mais au moins aussi précise que les précédentes (Alemu et al 2011), qui s'appuie, d'une part, sur la production globale des $\mathrm{AGV}$ à partir de la Matière Organique Fermentescible (MOF) et, d'autre part, sur l'influence du rapport entre les parois végétales (NDF) et la Matière Organique (MO) Digérées (MOD) (NDFD/MOD), ces deux critères ayant été souvent mesurés dans la littérature (ces dernières équations ont été calculées à partir de la base «Bovidig»). A partir des études conduites in vitro, des équations stœchiométriques ont également été proposées, les plus utilisées sont celles de Demeyer (1991) et de Blümmel et al (1999). Celles de Demeyer (1991) sont très cohérentes avec les données présentées dans les tableaux 1 et 2 .

Par ailleurs, pour raisonner la stœchiométrie des voies fermentaires du rumen et ses conséquences indépendamment des substrats et des régimes utilisés, on considère souvent les bilans d'éléments rapportés à chaque molécule d'AGV formée (tableau 2), voire même à l'unité de C sous forme d'AGV (Sauvant et Van Milgen 1995).

\section{4 / Les variations in vivo du profil fermentaire ruminal}

Dans un rumen qui fermente normalement et de façon stable, c'est-à-dire chez des animaux nourris avec des rations riches en fourrages, les proportions en AGV sont généralement considérées comme en moyenne de 66,19 et $11 \%$ pour les acides acétique, propionique et butyrique respectivement, les AGV mineurs représentant $4 \%$ environ du total des AGV. Le rapport acétate/propionate $(\mathrm{Ac} / \mathrm{Pr})$ de ce profil est d'environ 3,5 , ce qui correspond à un $\mathrm{pH}$ moyen du jus de rumen de 6,2 (Sauvant et Peyraud 2010), et est obtenu pour une ration présentant une teneur en NDF de 40 à $45 \%$ de la MS et en concentré de 10 à $25 \%$ de la MS. Ces mêmes auteurs estiment que le rumen a un fonctionnement normal lorsque le rapport $\mathrm{Ac} / \mathrm{Pr}$ est supérieur à 3 . A ce «profil de référence» des AGV, correspond en moyenne $2,53 \mathrm{~mol} \mathrm{C} / \mathrm{mol} \mathrm{AGV}$ et un poids moléculaire moyen de $67,5 \mathrm{~g}$. Les bilans en éléments de ce profil de référence sont présentés au tableau 2. 
Tableau 2. Bilans stœchiométriques des principales voies fermentaires en moles rapportées à une mole d'AGV formée.

\begin{tabular}{|c|c|c|c|c|c|c|c|c|}
\hline $\begin{array}{c}\text { Acide gras } \\
\text { volatil ou profil } \\
\text { d'AGV }\end{array}$ & $\begin{array}{c}\text { Nombre } \\
\text { de C }\end{array}$ & $\begin{array}{c}\mathrm{CO}_{2} \\
\text { fermentaire }\end{array}$ & $\mathrm{H}_{2}$ & $\underset{\text { potentiel }^{2}}{\mathrm{CH}_{4}}$ & $\begin{array}{l}\text { ATP } \\
\text { sans } \\
\mathrm{CH}_{4}{ }^{3} \\
\end{array}$ & $\begin{array}{l}\text { ATP } \\
\text { avec } \\
\mathrm{CH}_{4}^{4} \\
\end{array}$ & $\begin{array}{c}\text { ATP avec } \\
\mathrm{CH}_{4} / \mathrm{C} \\
\text { d'AGV }^{5}\end{array}$ & $\begin{array}{l}\mathrm{CO}_{2} \\
\text { total }^{6}\end{array}$ \\
\hline Acétate & 2 & 1 & 2 & 0,5 & 2 & 2,5 & 1,25 & 2 \\
\hline Propionate & 3 & 0 & -1 & $-0,25$ & 1,5 & 1,25 & 0,42 & 1 \\
\hline Butyrate & 4 & 2 & 2 & 0,5 & 3 & 3,5 & 0,88 & 3 \\
\hline Mineurs & 5 & 1 & -1 & $-0,25$ & 1 & 0,75 & 0,15 & 2 \\
\hline $\begin{array}{c}\text { Profil de } \\
\text { Référence }\end{array}$ & 2,53 & 0,92 & 1,31 & 0,32 & 1,97 & 2,30 & 0,91 & 1,92 \\
\hline Ac/Pr faible ${ }^{8}$ & 2,80 & 0,73 & 0,68 & 0,17 & 1,87 & 2,04 & 0,72 & 1,73 \\
\hline $\mathrm{Ac} / \mathrm{Pr}$ fort $^{9}$ & 2,31 & 0,93 & 1,54 & 0,38 & 1,98 & 2,37 & 1,02 & 1,92 \\
\hline
\end{tabular}

${ }^{1} \mathrm{CO}_{2}$ potentiellement formé par les fermentations, compte tenu des équations stœchiométriques citées au tableau 1 et en supposant qu'il n'y a pas de $\mathrm{CH}_{4}$ formé.

2 Potentiel car le calcul suppose que tous les $\mathrm{H}_{2}$ sont transformés en $\mathrm{CH}_{4}$.

3 ATP produit sans prendre en compte la formation de $\mathrm{CH}_{4}$.

4 ATP produit en intégrant la production potentielle de $\mathrm{CH}_{4}$.

5 Comme (4) mais rapporté à l'unité de C.

6 Evaluation qui tient compte de la neutralisation des AGV formés : 1 mole d'AGV est neutralisée par une mole de bicarbonate.

7 Profil de référence d'un rumen à fonctionnement normal (rapport $\mathrm{Ac} / \mathrm{Pr}$ voisin de 3,5 , cf. figure 1).

8 Moyenne des 20 profils de la figure 1 présentant les rapports $A c / P r$ les plus faibles (moyenne $A c / P r=1,15$ ).

9 Moyenne des 20 profils de la figure 1 présentant les rapports $\mathrm{Ac} / \mathrm{Pr}$ les plus forts (moyenne $\mathrm{Ac} / \mathrm{Pr}=5,54$ ).

Le profil en AGV peut varier sensiblement comme le montre la figure 1, issue de la base «Bovidig». Le profil de référence évoqué n'est pas situé au centre des nuages de points puisqu'il correspond à un type donné de régime et de fonctionnement du rumen qui ne pose en général pas de problème et qui n'est donc pas très fréquemment étudié. A l'évidence, la variation la plus marquée de ce profil concerne la substitution entre les proportions d'acétate et propionate, substitution qui constitue un événement pivot du métabolisme fermentaire du rumen, d'où l'importance $\mathrm{du}$ rapport Ac/Pr comme indicateur de l'état métabolique fermentaire du rumen.

Pour apprécier plus précisément l'amplitude des variations de la stœchiométrie des fermentations, les 40 profils extrêmes de cette base, en termes de rapport $\mathrm{Ac} / \mathrm{Pr}$, ont été interprétés à l'aide des critères du tableau 2. Pour les 20 profils présentant les valeurs les plus faibles du rapport $(\mathrm{Ac} / \mathrm{Pr}=1,15)$, cas de régimes très riches en énergie fermentescible (Sauvant et Van Milgen 1995), il y a, par rapport au profil de référence, plus de $\mathrm{C}$ par $\mathrm{AGV}(2,80 \mathrm{~mol})$, et moins de gaz, d' $\mathrm{H}_{2}$, d'ATP et de $\mathrm{CH}_{4}$ formés par AGV (tableau 2). Ces

Figure 1. Variations du profil molaire des AGV (en \% de la somme des AGV) dans le jus de rumen des bovins.

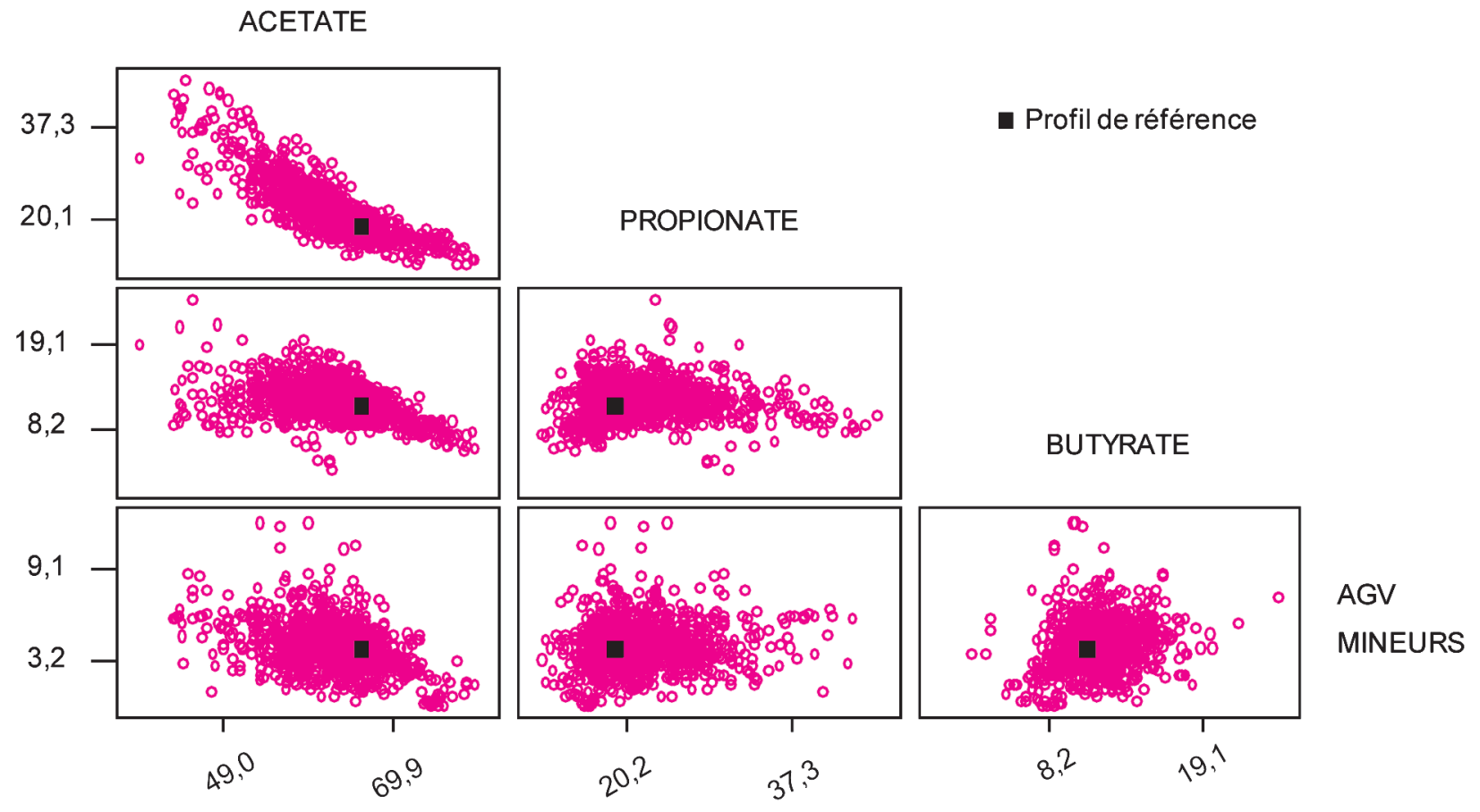

Base «Bovidig» 
Figure 2. Relation, calculée en intra-expérience, entre le rapport acétate/propionate du jus de rumen et l'énergie du $\mathrm{CH}_{4}$ exprimée en \% de l'énergie brute (données in vivo).

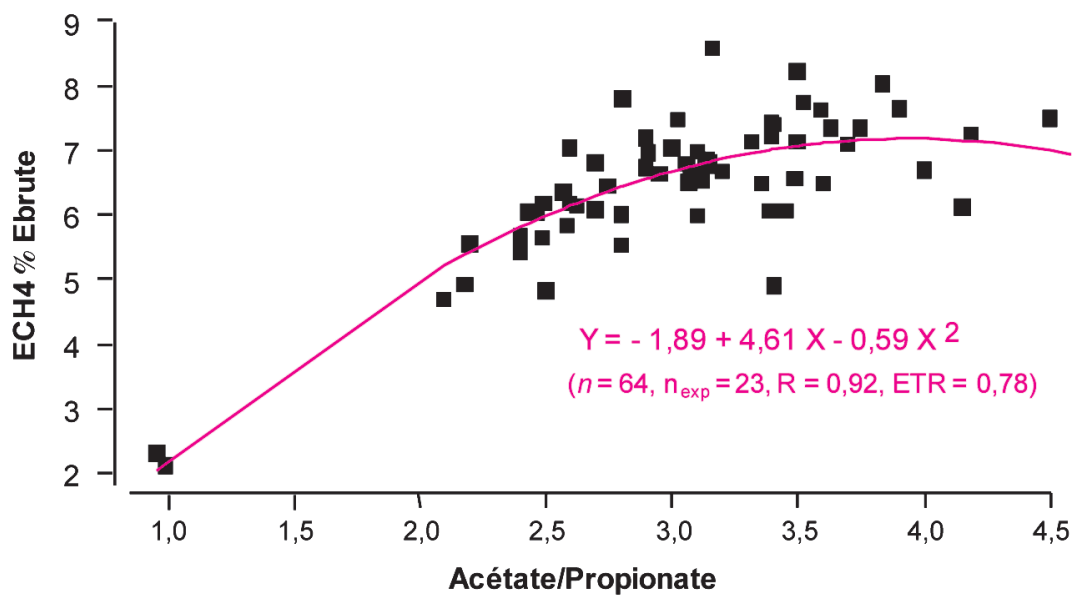

Base Rumener (UMR MOSAR 2011)

aspects traduisent un phénomène compensatoire inverse des fermentations intenses. En revanche, lorsque le rapport $\mathrm{Ac} / \mathrm{Pr}$ est très élevé $(\mathrm{Ac} / \mathrm{Pr}=5,54$ pour 20 rations), cas de régimes pauvres en énergie et produisant peu d'AGV, il y a, par rapport au profil de référence, moins de $\mathrm{C}$ par $\mathrm{AGV}$, à peu près le même gâchis de $\mathrm{C}$ en gaz, mais plus d' $\mathrm{H}_{2}$, d'ATP et de $\mathrm{CH}_{4}$ formés par mole d'AGV produit (tableau 2).

Lorsque ces différentes valeurs sont rapportées aux quantités de MOF, ou de glucose fermenté dans le rumen, plutôt qu'à celles d'AGV, les conclusions vont globalement dans le même sens. Ces deux profils extrêmes traduisent donc l'amplitude de l'adaptation des microorganismes à la disponibilité en énergie des substrats et soulignent clairement le rôle de la production de $\mathrm{CH}_{4}$ dans l'optimisation de l'utilisation de l'énergie des aliments.

Les relations entre profil fermentaire et production de $\mathrm{CH}_{4}$ se confirment in vivo. Ainsi, la figure 2 montre que le rapport $\mathrm{Ac} / \mathrm{Pr} \mathrm{du}$ jus de rumen est positivement lié au $\mathrm{CH}_{4}$ produit rapporté à la teneur en énergie brute du régime $\left(\mathrm{ECH}_{4} \% \mathrm{~EB}\right)$. Cette relation intra-expérience, extraite de 23 expériences (64 traitements) conduites sur bovins, ovins ou caprins, montre qu'un accroissement d'1 point du rapport $\mathrm{Ac} / \mathrm{Pr}$ est associé à une augmentation moyenne d'environ $1,5 \pm 0,4$ point de $\mathrm{ECH}_{4} \% \mathrm{~EB}$. En fait la relation est curvilinéaire (figure 2) et traduirait ainsi une certaine saturation de la méthanogenèse, ou bien l'existence de puits d' $\mathrm{H}_{2}$ concurrents à celle-ci, lorsque le rapport $\mathrm{Ac} / \mathrm{Pr}$ s'élève. Donc, le rapport Ac/Pr se répercute sur la méthanogenèse d'une façon d'autant plus marquée que le rapport $\mathrm{Ac} / \mathrm{Pr}$ est inférieur à 3. En effet, lorsque le rapport $\mathrm{Ac} / \mathrm{Pr}$ est inférieur
$\mathrm{C}_{16}$ ) synthétisés dans la mamelle à partir de l'acétate et du butyrate, soient liées à la production de $\mathrm{CH}_{4}$. Il est également à noter que, logiquement, compte tenu des relations entre le $\mathrm{pH}$ et le rapport $\mathrm{Ac} / \mathrm{Pr}$, les variations du $\mathrm{pH} d u$ rumen au sein d'une expérience sont étroitement et positivement liées à celles de la production de $\mathrm{CH}_{4}$. L'état d'acidose ruminale réduit donc la méthanogenèse :

$$
\begin{gathered}
\mathbf{C H}_{\mathbf{4}}(\mathbf{g} / \mathbf{k g M O D})=\mathbf{- 3 4 , 3}+\mathbf{1 0 , 3} \mathbf{~ p H} \\
\left(n=77, \mathrm{n}_{\exp }=13, \text { ETR }=5,9\right)
\end{gathered}
$$

\section{5 / Le bilan carbone des voies métaboliques fermentaires}

\section{a) Le $\mathrm{CO}_{2}$ issu des fermentations}

Pour le profil de référence des AGV, il y a environ $26 \%$ de $\mathrm{C}$ fermenté qui est perdu et il y a 0,92 mole de $\mathrm{CO}_{2}$ fermentaire (soit environ 23,6 L à la température du rumen, soit $39^{\circ} \mathrm{C}$ ) perdue par mole d'AGV formé (tableau 2). Ce tableau 2 précise que les fermentations aboutissant à des AGV à nombre pair de $\mathrm{C}$ sont associées à un "gâchis» de $\mathrm{C}$ sous forme de $\mathrm{CO}_{2}$. Elles peuvent apparaître ainsi moins efficaces puisqu'en théorie $1 / 3$ du flux de $\mathrm{C}$ empruntant les voies fermentaires serait ainsi perdu. En conséquence, le rapport Ac/Pr est une cause de variation importante de la proportion de $\mathrm{C}$ perdue sous forme gazeuse comme l'illustrent les deux dernières lignes du tableau 2. La figure 3 , issue de mesures et de calculs faits à partir de la base «Bovidig» montre l'amplitude des variations de la proportion de $\mathrm{C}$ dans les gaz par rapport au $\mathrm{C}$ dans les gaz et les $\mathrm{AGV}\left(\mathrm{C}_{\mathrm{gaz}}\left(\mathrm{C}_{\mathrm{gaz}}+\mathrm{C}_{\mathrm{AGV}}\right)\right)$, c'est-à-dire du gâchis de $\mathrm{C}$ fermenté, en fonction du rapport $\mathrm{Ac} / \mathrm{Pr}$ des $\mathrm{AGV}$. Ici encore, la relation est bien plus marquée lorsque le rapport $\mathrm{Ac} / \mathrm{Pr}$ est inférieur à 3 . nant que les teneurs en $A G$ pairs $\left(C_{4}\right.$ à
Figure 3. Influence du rapport acétate/propionate des AGV du jus de rumen sur la partition du carbone évaluée par la proportion de $C$ dans les gaz par rapport au $C$ dans les gaz et les $A G V\left(C_{g a z} /\left(C_{g a z} / C_{A G V}\right)\right)$.

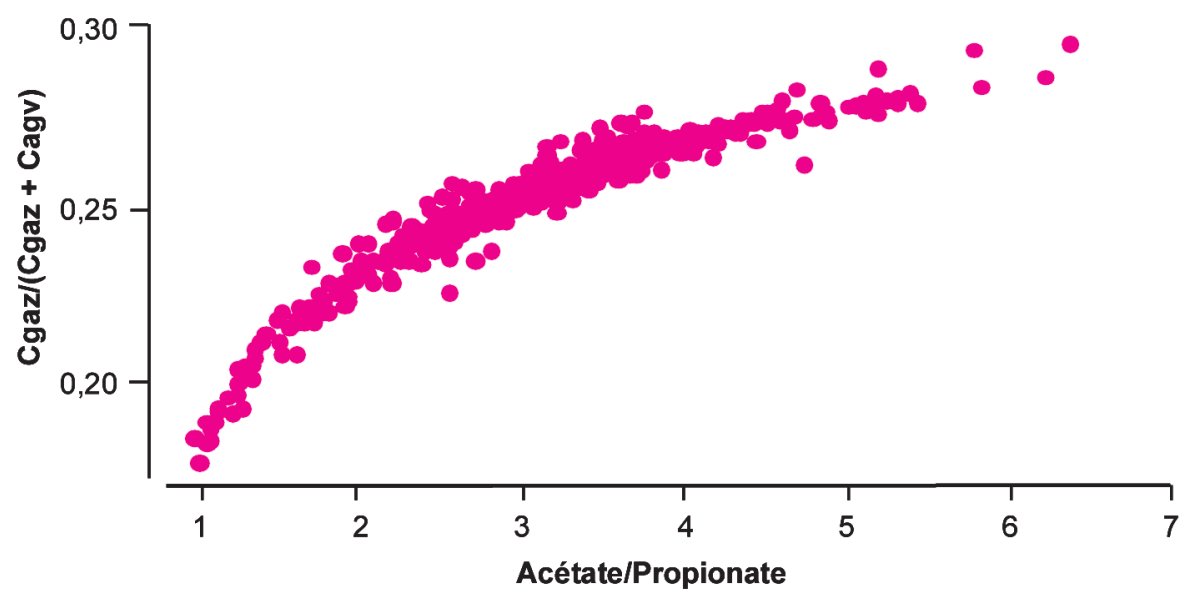

Base «Bovidig» 


\section{b) Le $\mathrm{CO}_{2}$ issu de la neutralisation}

Les différents AGV produits sont neutralisés, comme cela a été décrit par Meschy et al (2004). Ce sont principalement des molécules de bicarbonate $\left(\mathrm{HCO}_{3}^{-}\right)$, issues de la salive dont la sécrétion dépend de la mastication, qui neutralisent les AGV, le bilan de cette réaction est :

$$
\mathrm{HCO}_{3}{ }^{-}+\mathrm{H}_{3} \mathrm{O}^{+} \rightarrow 2 \mathrm{H}_{2} \mathrm{O}+\mathrm{CO}_{2}
$$

$\mathbf{A H}+\mathbf{H}_{2} \mathbf{O} \rightarrow \mathbf{A}^{-}+\mathbf{H}_{3} \mathbf{O}^{+}$(avec AH la forme acide des $\mathrm{AGV}$ et $\mathrm{A}^{-}$la forme basique)

Cette réaction n'est totale qu'à $\mathrm{pH}$ neutre et constant; on admet qu'elle est en moyenne réalisée à $90 \%$ dans le rumen. On peut donc dire que chaque molécule d'AGV neutralisée entraîne la formation de près d'une molécule de $\mathrm{CO}_{2}$ non fermentaire. En conséquence, au bilan global, chaque molécule d'AGV formé génère en tout de 1 à 3 molécules $(25,6$ à $76,8 \mathrm{~L})$ de $\mathrm{CO}_{2}$ total (tableau 2) dont une partie est transformée en $\mathrm{CH}_{4}$. In vitro, selon Blümmel et al (1999), le $\mathrm{CO}_{2}$ issu des tampons représenterait en moyenne jusqu'à 1,8 fois le $\mathrm{CO}_{2}$ issu des fermentations. Ces résultats montrent qu'une partie du $\mathrm{CH}_{4}$ produit peut provenir des bicarbonates du rumen utilisés pour la neutralisation des acides, ce qui constitue un aspect rarement évoqué dans la littérature.

A un niveau de l'écosystème du rumen, la partition du $\mathrm{C}$ issu des aliments est l'objet d'un intérêt croissant avec les approches «bilan carbone». On estime qu'en moyenne, pour $100 \mathrm{C}$ de substrat dégradé dans le rumen, on en retrouve 56 dans les $\mathrm{AGV}, 19$ dans les gaz et 25 dans les microorganismes (Sauvant et Giger-Reverdin 2009a). Ces proportions varient principalement en fonction de l'efficacité de la croissance microbienne et du profil en AGV.

\section{6 / Le métabolisme énergétique fermentaire}

La dégradation des glucides fournit de l'énergie sous forme d'ATP utilisé par les microorganismes (cf. § 1.7). Le profil de référence évoqué plus haut correspond à près de $2 \mathrm{~mol} \mathrm{ATP} / \mathrm{mol}$ $\mathrm{AGV}$, ce rapport varie largement selon les voies fermentaires (tableau 2). Ainsi, il apparaît que la prise en compte de la production de $\mathrm{CH}_{4}$ améliore nettement le rendement énergétique de la synthèse des $\mathrm{AGV}$ à nombre pair de $\mathrm{C}$, et réduit celui de la synthèse des $\mathrm{AGV}$ à nombre impair de C. Pour les 3 profils de mélange d'AGV (référence, rapport $\mathrm{Ac} / \mathrm{Pr}$ faible ou élevé, tableau 2), le rendement ATP/AGV est amélioré par la méthanogenèse. Par ailleurs, si on se rapporte à l'unité élémentaire d'énergie formée et mise à disposition de l'animal hôte, c'est-à-dire à l'unité de C d'AGV, le tableau 2 montre que le rapport ATP disponible/C d'AGV diminue fortement avec l'accroissement du nombre de $\mathrm{C}$ de la chaîne d'AGV (Sauvant et Van Milgen 1995). Cette hiérarchie nette montre bien qu'une relative carence en énergie des substrats vis-à-vis des microorganismes est principalement compensée par des fermentations acétiques, associées à une méthanogenèse marquée qui permet de maximiser la quantité d'énergie extraite et valorisée de ce substrat.

Les ATP formés sont utilisés par les microorganismes du rumen pour assurer leurs besoins d'entretien (environ 15 à 55 mol ATP par kg de MS Microbienne (MSM) et par jour selon les données de la littérature) et de croissance. Ce besoin de croissance correspond en moyenne à $15 \mathrm{~g}$ de $\mathrm{MSM} / \mathrm{mol}$ ATP formé (coefficient souvent noté YATP), cette valeur varie entre 10 et 30 selon les études (Belaich 1986). Il y a donc deux grandes étapes à considérer dans l'utilisation de l'énergie par les microorganismes : la production des ATP par les fermentations et leur utilisation pour soutenir l'activité microbienne. Certains systèmes d'unités d'alimentation distinguent ces deux étapes. Lorsqu'on combine ces deux rendements partiels en les multipliant, on aboutit approximativement à un rendement moyen de 50 à 90 g de MSM par mole de glucose fermenté, soit environ 0,3 à $0,5 \mathrm{~g}$ de MSM par g de glucose fermenté. Rappelons que, dans le cas du système PDI, le rendement de $145 \mathrm{~g}$ de matières azotées microbiennes formées par kg de MOF correspond approximativement à $0,3 \mathrm{~g} \mathrm{MSM} / \mathrm{g} \mathrm{MOF}$ de glucose, soit une hypothèse assez basse d'efficacité de la croissance microbienne.

\section{7 / Le métabolisme de l'hydro- gène et la production de métha- ne}

Le tableau 1 indique que des quantités non négligeables d'hydrogène moléculaire sont générées à travers les réactions fermentaires, cet hydrogène est associé à des molécules spécifiques de transport (le couple $\mathrm{NADH}_{2} \Leftrightarrow \mathrm{NAD}+$ $\mathrm{H}_{2}$ étant le plus connu). Ce surplus potentiel d'hydrogène doit être utilisé à travers différentes voies métaboliques, appelées puits d'hydrogène, résumées dans la figure 4.

Cette figure, et les tableaux 1 et 2, permettent de souligner que les voies fermentaires des $\mathrm{AGV}$ à nombre pair de C (acétate et butyrate) produisent des excès importants $\mathrm{d}^{\prime} \mathrm{H}_{2}$ alors que celles conduisant aux $\mathrm{AGV}$ à nombre de $\mathrm{C}$ impair (propionate) sont, au contraire, utilisatrices d' $\mathrm{H}_{2}$. De ce fait, le rapport $\mathrm{Ac} / \mathrm{Pr}$ est aussi un indicateur important du métabolisme de l'hydrogène dans le rumen. La sortie principale et logique de l'hydrogène moléculaire est la forme $\mathrm{d}^{\prime} \mathrm{H}_{2}$ gazeux. Cependant, les conditions thermodynamiques du milieu ruminal ne sont pas favorables, car il n'est pas assez acide et son potentiel redox n'est pas assez négatif. En pratique, la teneur en hydrogène gazeux dissous du jus de rumen est très faible $(0,1$ à moins de $50 \mu \mathrm{M})$, alors que le niveau de saturation serait d'environ $735 \mu \mathrm{M}$. Cet écart important traduit un flux d'utilisation intense de $\mathrm{H}_{2}$ moléculaire qui serait principalement lié à son utilisation pour produire du $\mathrm{CH}_{4}$, sachant que la croissance et l'activité des Archaea méthanogènes dépendent de la teneur en $\mathrm{H}_{2}$ du milieu selon une loi de Monod avec une constante située vers 5-10 $\mu \mathrm{M}$ (Janssen 2010). In vitro, $48 \%$ de $\mathrm{H}_{2}$ serait impliqué dans l'élaboration du $\mathrm{CH}_{4}$ contre $33 \%$ dans la synthèse des $\mathrm{AGV}$ impairs, $12 \%$ dans les synthèses microbiennes et de l'ordre de $2 \%$ dans

Figure 4. Les principales voies métaboliques de production et d'utilisation de l'hydrogène dans le rumen.

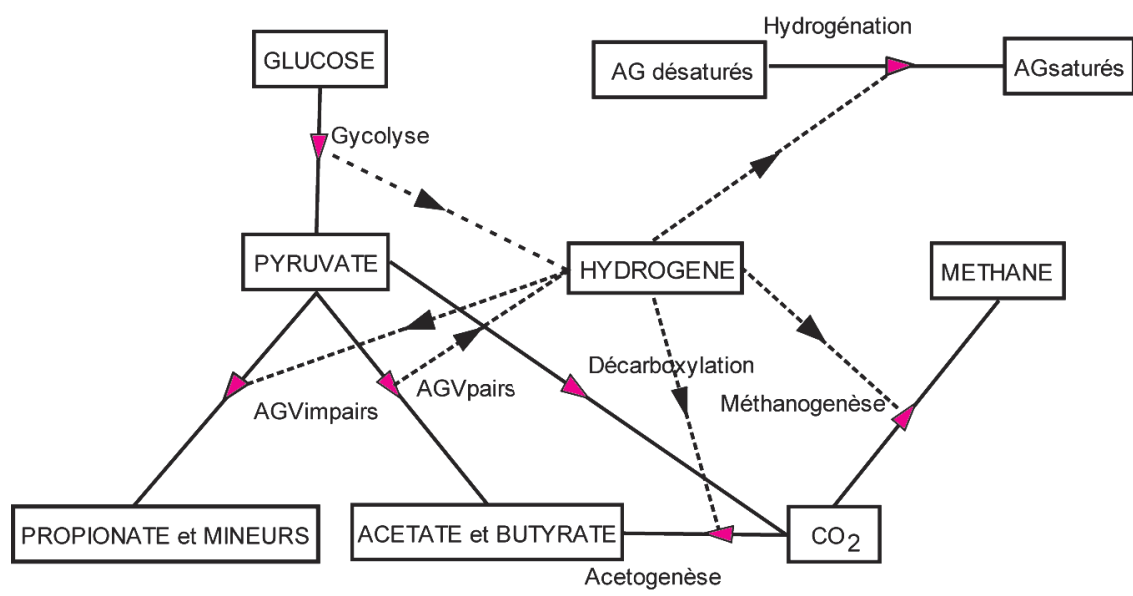


les saturations des AG (Czerkawzki 1986). Cependant un débat demeure sur la proportion d' $\mathrm{H}_{2}$ disponible qui serait impliquée dans la production de $\mathrm{CH}_{4}$. Elle est de $100 \%$ dans les modèles stœchiométriques proposés (cf. § 3.2). Des résultats in vitro récents de Serment et al (communication personnelle) suggèrent que cette proportion pourrait varier en fonction des relations stœchiométriques appliquées, du niveau de disponibilité en $\mathrm{H}_{2}$ et de l'existence évoquée de puits d' $\mathrm{H}_{2}$.

\section{2 / Influences des princi- paux facteurs alimentaires sur les fermentations et la production de méthane}

\section{1 / Les modes d'expression de la production de $\mathrm{CH}_{4}$}

Dans la littérature, la production du méthane est exprimée dans des unités très variables : en énergie ( $k$ cal ou $M J)$, en volume ou en masse. De plus, la base d'expression varie très largement, ainsi, le $\mathrm{CH}_{4}$ produit peut être exprimé par animal, ou par $\mathrm{kg}$ de poids vif pour pouvoir comparer bovins, ovins et caprins entre eux (Sauvant et al 2006), ou par unité de produit élaboré ( $\mathrm{kg}$ de lait, carcasse, viande...). Actuellement, le $\mathrm{CH}_{4}$ produit est de plus en plus souvent exprimé par unité d'intrant. Dans ce cas, plusieurs unités existent dans la littérature (valeurs observées dans la base «Rumener») : Energie brute $\left(\mathrm{ECH}_{4} \% \mathrm{~EB}=6,68 \pm 1,83, n=\right.$ $1105), \mathrm{kg}$ de $\mathrm{MS}$ ingérée (MSI) $\left(\mathrm{CH}_{4}\right.$, $\mathrm{g} / \mathrm{kgMS})=22,5 \pm 6,8, n=1091)$, ou $\mathrm{kg}$ MOD ingérée $\left(\mathrm{CH}_{4}, \mathrm{~g} / \mathrm{kgMOD}\right)=34,1$ $\pm 9,2, n=1085$ ) ou, beaucoup plus rarement en raison des difficultés de mesure, MOD dans le rumen (apparente ou réelle). Des arguments sur le choix entre ces unités sont apportés dans le $\S 2.2$ ci-dessous. Les critères rapportés à l'intrant sont statistiquement très liés entre eux, par exemple, pour la base «Rumener», la relation globale entre $\mathrm{CH}_{4}$ en $\mathrm{g} / \mathrm{kgMS}$ et $\mathrm{ECH}_{4} \% \mathrm{~EB}$ est :

$$
\begin{gathered}
\mathbf{C H}_{\mathbf{4}}(\mathbf{g} / \mathbf{k g M S})=\mathbf{0 , 3 4}+\mathbf{3 , 2 6} \mathbf{E C H}_{\mathbf{4}} \% \mathbf{E B} \\
\left(n=1104, \mathrm{R}^{2}=0,98, \mathrm{ETR}=0,84\right)
\end{gathered}
$$

et celle entre $\mathrm{CH}_{4}$ en $\mathrm{g} / \mathrm{kgMOD}$ et $\mathrm{CH}_{4}$ en $\mathrm{g} / \mathrm{kgMS}$ est :

$$
\begin{gathered}
\mathbf{C H}_{\mathbf{4}}(\mathbf{g} / \mathbf{k g M O D})=\mathbf{4 , 6 8}+\mathbf{1 , 3 2} \mathbf{C H}_{\mathbf{4}} \\
(\mathbf{g} / \mathbf{k g M S}) \\
\left(n=1077, \mathrm{R}^{2}=0,80, \mathrm{ETR}=4,09\right)
\end{gathered}
$$

Ces relations sont encore plus précises lorsqu'elles sont calculées en intraexpérience.

Cette diversité d'unités et surtout de modes d'expression de la production de
$\mathrm{CH}_{4}$ peut soulever la question de l'interprétation des résultats publiés. En effet, selon l'expression, des conclusions peuvent être contradictoires ou prêter à une interprétation erronée, voire risquée. Par exemple, les ruminants dont le potentiel de production est faible à moyen et recevant une ration pauvre produisent bien moins de $\mathrm{CH}_{4}$ par animal que les ruminants à potentiel de production élevé et alimentés avec des rations riches. En revanche, ils produisent plus de $\mathrm{CH}_{4}$, exprimé par unité de produit ou bien de quantités ingérées Dans un tel contexte, il convient d'être très prudent au niveau des conclusions à tirer sur la production de $\mathrm{CH}_{4}$, par exemple entre les pays tempérés et tropicaux

\section{2 / Le rôle pivot de la teneur en Matière Organique Digestible (MOD)}

Compte tenu du fait que le $\mathrm{CH}_{4}$ produit est d'abord lié à la MO qui fermente dans le rumen (MOF), il est logique de chercher à l'exprimer en fonction d'un paramètre qui soit à la fois $i$ ) fréquemment mesuré, donc présent dans les tables des valeurs des aliments ou dans les publications, ii) facilement interprétable en termes de teneur en énergie (UFL ou UFV) et iii) très lié à la MOF. La teneur du régime en MOD $(=\mathrm{MO} \times \mathrm{dMO})$ répond a priori le mieux à ce cahier des charges et constitue donc un mode d'expression à privilégier. En effet, elle détermine étroitement les teneurs en UF des régimes (Baumont et al 2007), elle est statistiquement assez bien liée à la teneur en MOF dans le rumen (Sauvant et GigerReverdin 2009b), enfin elle est bien reliée à la production de $\mathrm{CH}_{4}$ exprimée sur la MS :

$$
\begin{gathered}
\mathbf{C H}_{\mathbf{4}} / \mathbf{M S I}(\mathbf{g} / \mathbf{k g M S})=\mathbf{7 , 1 4}+\mathbf{0 , 2 2} \text { MOD } \\
(\mathbf{\% M S}) \\
\left(n=976, \mathrm{n}_{\exp }=170, \mathrm{R}^{2}=0,81\right. \\
\left.\operatorname{ETR}^{2}=2,7\right)[\mathbf{1}]
\end{gathered}
$$

ou par rapport au poids vif, pour pouvoir associer les différentes espèces de ruminants dans une même relation (Sauvant et al 2006) :

$$
\begin{gathered}
\mathbf{C H}_{\mathbf{4}}(\mathbf{g} / \mathbf{k g P V})=\mathbf{0 , 0 8 3}+\mathbf{0 , 0 2 5} \text { MODI } \\
(\mathbf{g} / \mathbf{k g P V}) \\
\left(n=1008, \mathrm{n}_{\exp }=172, \mathrm{R}^{2}=0,90,\right. \\
\text { ETR }=0,05) \quad[\mathbf{1 b i s}]
\end{gathered}
$$

Ces deux dernières régressions, calculées en intra-expérience, peuvent être utilisées en pratique, pour prévoir simplement les quantités de $\mathrm{CH}_{4}$ attendues pour une teneur ou une ingestion moyenne de MOD. Elles sont plus précises que les équations comparables basées uniquement sur la Matière Sèche (MS). Les coefficients des pentes indi- quent qu'en moyenne, $22 \pm 2$ et $25 \pm 1 \mathrm{~g}$ (valeurs non significativement différentes) de $\mathrm{CH}_{4}$ sont produits par kg MOD. En revanche, compte tenu du fait que la constante est positive et différente de 0 , ces deux valeurs de pente sont plus faibles que la valeur moyenne citée plus haut de $34 \mathrm{~g}$ de $\mathrm{CH}_{4}$ par $\mathrm{kg}$ de MOD.

Au-delà de ces deux relations $\mathrm{CH}_{4}=$ $\mathrm{f}(\mathrm{MOD})$, le rapport $\mathrm{CH}_{4} / \mathrm{MOD}(\mathrm{g} / \mathrm{kg})$ représente le $\mathrm{CH}_{4}$ produit par unité de MOD et permet donc de préciser les valeurs fournies par les équations [1] et [1bis]. Ce sera donc le critère le plus considéré dans la suite de cet article, il permet de décomposer la production de $\mathrm{CH}_{4}$ par kg de MS, ou par animal, entre les effets globaux liés au niveau de MOD et de la nature de la MOD. Ainsi, pour l'expression par rapport à la MS on a :

\section{$\mathrm{CH}_{4} / \mathrm{MS}=[(\mathrm{MO} * \mathrm{dMO}) / \mathrm{MS}] * \mathrm{CH}_{4} / \mathrm{MOD}$}

Sachant que «par construction», en raison des valeurs non nulles des constantes des régressions [1] et [1bis], il y a une relation négative entre les teneurs en MOD des aliments et le critère $\mathrm{CH}_{4} / \mathrm{MOD}$, donc plus un aliment ou une ration est digestible, moins elle produit de $\mathrm{CH}_{4}$ par unité de MOD. Par exemple, l'équation [1] peut s'écrire sous la forme :

\section{$\mathrm{CH}_{4} / \mathrm{MOD}=0,22+(7,14 / \mathrm{MOD} \% \mathrm{MS})$}

Ce qui aboutit, par exemple, à des valeurs décroissantes de 39,8 à 30,9 $\mathrm{g} / \mathrm{kgMOD}$ lorsque MOD s'accroît de 40 à $80 \% \mathrm{MS}$ respectivement. Ces relations incontournables créent des difficultés pour prévoir avec précision, à partir de critères simples, la production de $\mathrm{CH}_{4}$. Elles sont principalement dues aux relations entre les profils en AGV dans le rumen et la teneur en MOD\%MS des aliments et des rations. En effet, les rations pauvres en MOD\%MS présentent des fermentations ruminales à rapport $\mathrm{Ac} / \mathrm{Pr}$ élevées et sont donc plus méthanogènes.

\section{3 / Influence du niveau d'ali- mentation}

\section{a) Sur les transits et la digestibilité}

L'influence négative du Niveau Alimentaire (NA), calculé comme la MS Ingérée (MSI) exprimée en \% du PV (MSI\%PV), sur la production de $\mathrm{CH}_{4}$ exprimée par rapport à l'énergie brute des rations ingérées $\left(\mathrm{ECH}_{4} \% \mathrm{~EB}\right)$ a été précisée récemment à partir des données de la base «Rumener» (Sauvant et Giger-Reverdin 2009b). Cette base a été utilisée pour dégager des relations exprimées en $\mathrm{gCH}_{4} /$ $\mathrm{kgMOD}$. Une sous-base des expérien- 
Figure 5. Influence, calculée en intra-expérience, du niveau d'ingestion de MS sur le rapport acétate/propionate dans le rumen.

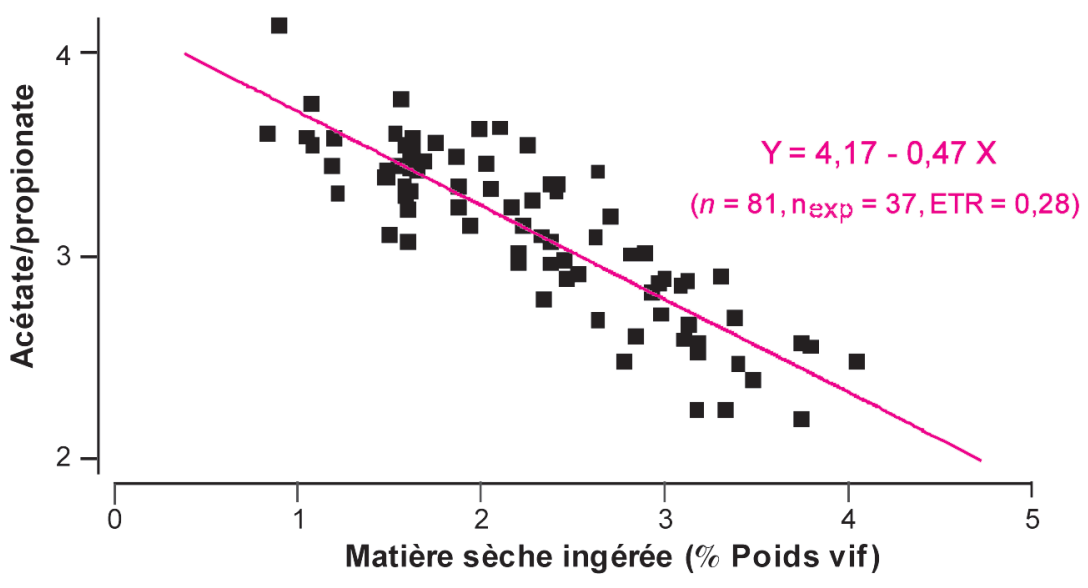

Base «Bovidig», expériences focalisées sur le niveau d'ingestion ces ciblées sur les influences du NA (103 expériences, 295 traitements) a été extraite de la base «Rumener». Il est connu que les transits des particules et des liquides sont accrus avec l'augmentation du NA, qui réduit de ce fait la durée de séjour et la digestion microbienne des substrats dans le rumen. En conséquence, le NA influence négativement la digestibilité des rations (Sauvant et Giger-Reverdin 2009b). Pour la teneur en MOD $(\mathrm{g} / \mathrm{kgMS})$ des rations, la relation intra-expérience est la suivante :

$$
\begin{gathered}
\text { MOD }(\mathbf{g} / \mathbf{k g} \text { MS })=700-22,7 \text { MSI\%PV } \\
\left(n=292, \mathrm{n}_{\exp }=94, \mathrm{ETR}=16\right)
\end{gathered}
$$

b) Sur les profils fermentaires, la disponibilité en hydrogène et la production de $\mathrm{CH}_{4}$

Les variations du niveau d'alimentation influencent le profil en AGV du rumen. A partir de la base «Bovidig» et des comparaisons portant uniquement sur le NA, à ration égale, il apparaît une baisse systématique du rapport $\mathrm{Ac} / \mathrm{Pr}$ comme le montre la figure 5 et l'équation [3] :

$$
\begin{array}{r}
\text { Ac/Pr }=4,17-\mathbf{0 , 4 7} \text { MSI\%PV } \\
\left(n=81, \mathrm{n}_{\text {exp }}=37, \text { ETR }=0,28\right)
\end{array}
$$

D'après cette régression, le rapport $\mathrm{Ac} / \mathrm{Pr}$ devient inférieur à 3 lorsque NA est supérieur à 2,5.

Avec la base «Rumener», en regroupant des expériences focalisées sur l'influence du NA d'un même régime, Sauvant et Giger-Reverdin (2009b) ont montré que le NA réduisait significativement la valeur du rapport $\mathrm{ECH}_{4} \%$ Ebrute, donc $\mathrm{CH}_{4} / \mathrm{MSI}$ qui lui est très lié. Il en est de même pour le rapport $\mathrm{gCH}_{4} / \mathrm{kgMOD} \mathrm{:}$
Figure 6. Influence, calculée en intra-expérience, du niveau d'alimentation sur la production de $\mathrm{CH}_{4}$ en $\mathrm{g}$ par $\mathrm{kg}$ de $\mathrm{MOD}$.

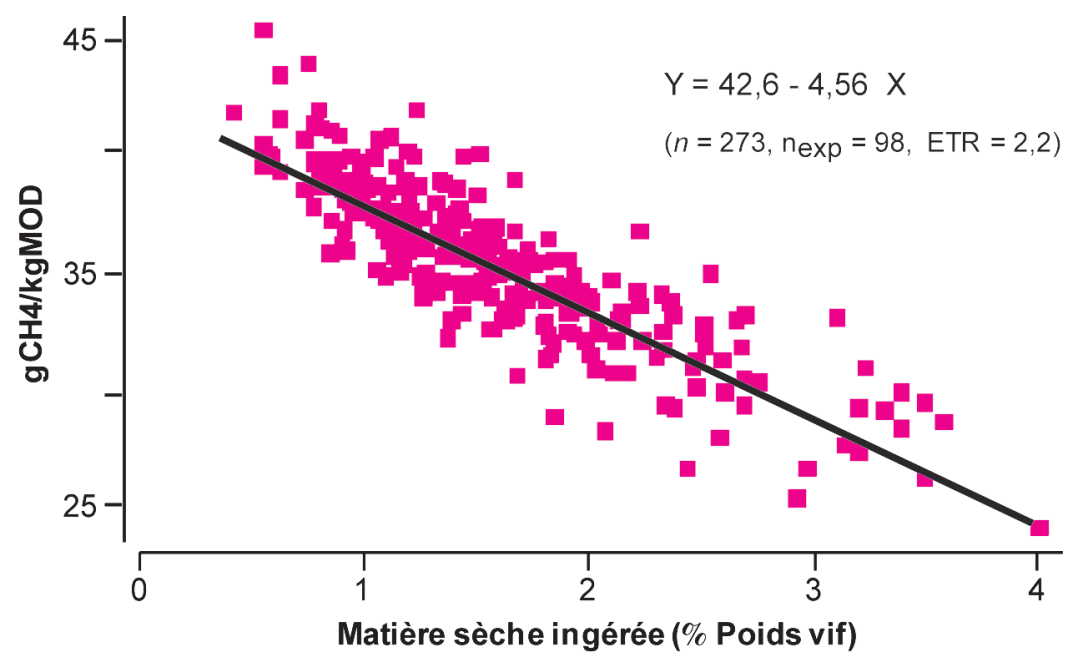

Base «Rumener», UMR MoSAR expériences focalisées sur le niveau d'ingestion
$\mathrm{CH}_{\mathbf{4}}(\mathbf{g} / \mathbf{k g}$ MOD $)=\mathbf{4 2 , 6}-\mathbf{4 , 5 6} \mathrm{MSI} \% \mathrm{PV}$
$\left(n=273, \mathrm{n}_{\exp }=98, \mathrm{ETR}=2,2\right)$

Cette relation est présentée à la figure 6 .

$\mathrm{Si}$ la production de $\mathrm{CH}_{4}$ est rapportée à la MSI $(\mathrm{g} / \mathrm{kg})$, la relation est assez comparable :

$$
\begin{gathered}
\mathbf{C H}_{\mathbf{4}}(\mathbf{g} / \mathbf{k g} \mathbf{M S})=\mathbf{2 9 , 5}-\mathbf{3 , 8 8} \mathbf{M S I} \% \mathbf{P V} \\
\left(n=273, \mathrm{n}_{\exp }=98, \mathrm{ETR}=1,6\right)
\end{gathered}
$$

Il apparaît donc une assez bonne cohérence entre les équations [3] et [4], elles traduisent globalement un ratio d'équivalence $\quad\left(\mathrm{CH}_{4} / \mathrm{MOD}\right) /(\mathrm{Ac} / \mathrm{Pr})$ d'environ 10.

\section{4 / Influences de la qualité du fourrage}

Cette question mérite un développement particulier pour différentes raisons : les ruminants sont les animaux qui valorisent le mieux les fourrages et le concept de qualité des fourrages agrège les aspects liés à l'ingestion et à la digestibilité au sein de la valeur alimentaire qui peut être exprimée en MODI (g/kgPV).

\section{a) Sur la digestibilité}

Les tables INRA 2007, comme les éditions précédentes, ont largement montré que la teneur en MOD des fourrages dépendait étroitement du stade de développement des plantes, donc de leur teneur en constituants pariétaux. Ainsi, à partir tables INRA (2007), la relation moyenne calculée entre MOD ( $\mathrm{g} / \mathrm{kgMS})$ et NDF ( $\mathrm{g} / \mathrm{kgMS})$ est :

$$
\begin{gathered}
\text { MOD }(\mathbf{g} / \mathbf{k g} \mathbf{M S})=\mathbf{1 0 5 2}-\mathbf{0 , 8 3} \text { NDF } \\
(\mathbf{g} / \mathbf{k g M S}) \\
(n=1140, \text { ETR }=32)
\end{gathered}
$$

Avec des coefficients correcteurs de la constante : $+24,5$ pour les prairies permanentes, $+45,5$ pour les graminées et 0 pour les légumineuses.

b) Sur la production de $\mathrm{CH}_{4}$ et les profils fermentaires

Les traitements de la base «Rumener» qui correspondent à des régimes constitués à $100 \%$ de fourrages ont été considérés. Pour préciser le rôle de la MOD les critères sont exprimés d'abord par rapport à la MS. En intra-expérience, la production de $\mathrm{CH}_{4}(22,3 \pm 5,8 \mathrm{~g} / \mathrm{kgMS}, \min =$ $6,8 ; \max =36,1)$ est négativement et étroitement liée au NA et curvilinéairement à la teneur en MOD $(612 \pm 78$ $\mathrm{g} / \mathrm{kgMS}, \min =407, \max =797$ ) du fourrage selon l'équation :

$$
\begin{gathered}
\mathrm{CH}_{4}(\mathrm{~g} / \mathrm{kg} \mathrm{MS})=-22,4-2,25 \mathrm{MSI} \% \mathrm{PV}+ \\
\mathbf{0 , 1 3 7} \text { MOD }(\mathbf{g} / \mathbf{k g M S})-\mathbf{0 , 0 0 0 0 9} \mathrm{MOD}^{2} \\
(\mathbf{g} / \mathbf{k g M S}) \\
\left(n=283, \mathrm{n}_{\exp }=53, \mathrm{ETR}=1,6\right)[5]
\end{gathered}
$$


Figure 7. Relation, calculée en intra-expérience, entre la teneur en MOD des fourrages et la production de $\mathrm{CH}_{4}$ associée.

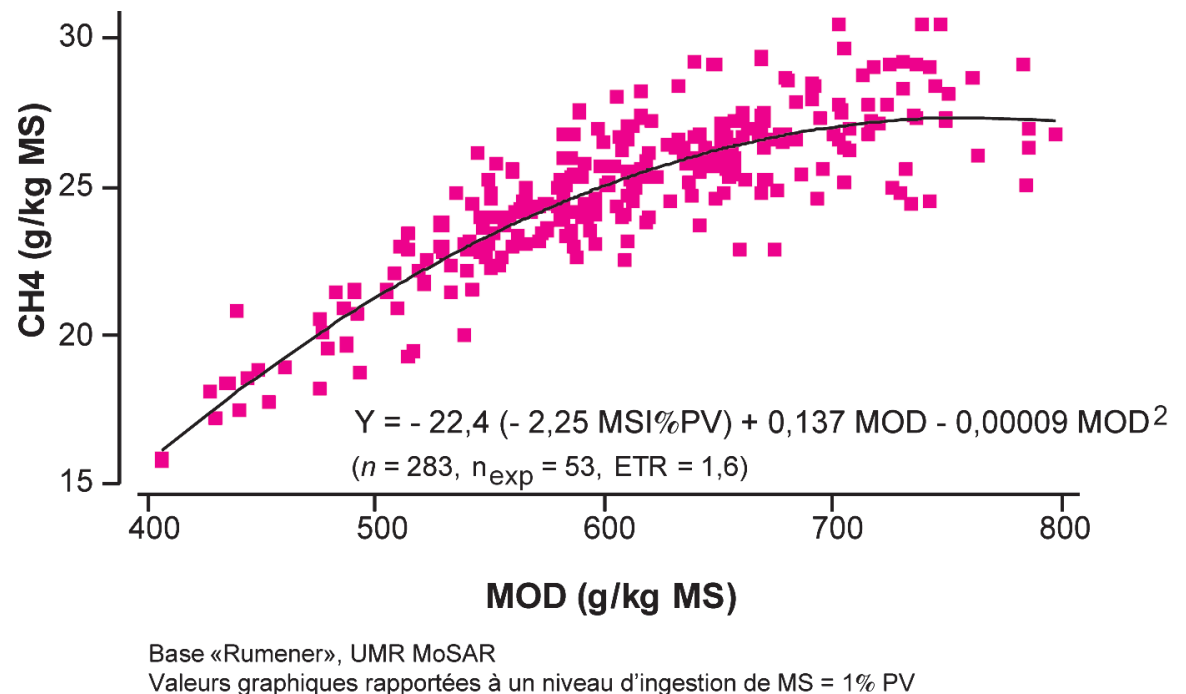

L'équation [5] peut être appliquée pour estimer la production potentielle de $\mathrm{CH}_{4}$ associée aux fourrages des tables utilisés comme seul aliment d'une ration à partir des valeurs moyennes de MOD et d'ingestibilité potentielle. La figure 7 indique l'allure curvilinéaire de cette relation, calculée pour un niveau alimentaire correspondant à une ingestion de MS représentant $1 \%$ du poids vif. Selon cette relation, pour des fourrages pauvres $(\mathrm{MOD}=400$ à 450 $\mathrm{g} / \mathrm{kgMS}$ ), la réponse marginale au $\mathrm{g}$ d'accroissement de MOD $\left(\mathrm{dCH}_{4} /\right.$ dMOD) est importante (environ 60 $\mathrm{gCH}_{4} / \mathrm{kgMOD}$ ). Pour ces fourrages la production moyenne de $\mathrm{CH}_{4}$ est de l'ordre de $40 \mathrm{~g} / \mathrm{kgMOD}$. Pour de meilleurs fourrages la réponse marginale diminue jusqu'à 0 pour des teneurs en MOD de $760 \mathrm{~g} / \mathrm{kgMS}$ qui correspondent alors à une production moyenne de $\mathrm{CH}_{4}$ de l'ordre de $36 \mathrm{~g} / \mathrm{kgMOD}$.

Les fourrages les plus pauvres produisent donc, en moyenne, plus de $\mathrm{CH}_{4}$ par $\mathrm{kg}$ de $\mathrm{MOD}$, mais moins de $\mathrm{CH}_{4}$ par $\mathrm{kg}$ de MS que les fourrages les plus riches. Cette apparente contradiction entre modes d'expression de la production du $\mathrm{CH}_{4}$ reflète les différences de teneur en MOD et de profil fermentaire entre les fourrages pauvres et les fourrages riches. Elle souligne la nécessité d'exprimer la production de $\mathrm{CH}_{4}$ selon les deux modes d'expression, ce qui est de plus en plus pratiqué dans les publications. Cette différence selon le mode d'expression n'a pas été observée dans le travail de comparaison récent d'Archimède et al (2011) entre fourrages tropicaux et tempérés. Cela pourrait s'expliquer en partie par l'influence des métabolites secondaires présents dans certains fourrages tropicaux sur la production de $\mathrm{CH}_{4}$ (cf. Popova et al 2011) qui n'a pas été totalement prise en compte dans cette étude.

\section{5 / Influences de l'apport de concentré}

\section{a) Sur la digestibilité}

Un apport de concentré permet toujours d'améliorer la digestibilité de la MO ou de l'énergie d'un régime. Cependant, la réponse dépend notamment de la qualité du fourrage considéré, du niveau de concentré, des interactions digestives ainsi que du niveau alimentaire comme l'ont montré Sauvant et Giger-Reverdin (2009b).

b) Sur les profils fermentaires et la production de $\mathrm{CH}_{4}$

Avec la base «Bovidig», en ne considérant que les expériences focalisées sur l'effet du niveau de concentré (PCO exprimé en proportion de la MS du régime, de manière à aboutir à des coefficients de régression simples), il apparaît une influence quadratique de la
Base «Bovidig»
Proportion de Concentré (PCO) sur le rapport acétate/propionate.

$$
\begin{aligned}
& \mathrm{Ac} / \mathrm{Pr}=3,48+0,646 \mathrm{PCO}-\mathbf{2 , 7 6} \mathbf{P C O}^{2} \\
& \left(n=325, \mathrm{n}_{\text {exp }}=139, \mathrm{ETR}=0,28\right)
\end{aligned}
$$

C'est surtout le terme quadratique qui est marquant (maximum de $\mathrm{Ac} / \mathrm{Pr}$ pour PCO $=0,12)$, montrant ainsi que les effets négatifs du concentré deviennent dominants à mesure que la proportion s'accroît au-delà d'un seuil d'environ 0,4 comme l'illustre la figure 8 .

Sur le rapport $\mathrm{ECH}_{4} \%$ Ebrute, il a été montré depuis assez longtemps (GigerReverdin et al 2000) une influence curvilinéaire du niveau de concentré, ce qui a été confirmé par Sauvant et GigerReverdin (2009b). L'influence de PCO est aussi curvilinéaire pour le rapport $\mathrm{CH}_{4} / \mathrm{MOD}$ :

$$
\begin{gathered}
\mathrm{CH}_{4}(\mathrm{~g} / \mathbf{k g} \mathrm{MOD})=\mathbf{3 5 , 3}+\mathbf{1 5 , 8 4} \mathbf{P C O} \\
-\mathbf{3 4 , 5 9} \mathbf{P C O}^{2} \\
\left(n=290, \mathrm{n}_{\mathrm{exp}}=97, \mathrm{ETR}=3,5\right) \quad \text { [7] }
\end{gathered}
$$

La figure 9 présente l'allure de cette relation, la production maximale de $\mathrm{CH}_{4}(37,1 \mathrm{~g} / \mathrm{kg}$ MOD) est réalisée pour $\mathrm{PCO}=0,23$. En deçà elle varie peu et, au-delà, la production de $\mathrm{CH}_{4}$ décroît quand PCO augmente. Il y a donc une assez bonne cohérence entre les conséquences de l'apport de concentré sur les profils fermentaires et le $\mathrm{CH}_{4}$ produit (figures 8 et 9) avec, comme pour l'effet de MSI\%PV, un ratio d'équivalence $\left(\mathrm{CH}_{4} / \mathrm{MOD}\right) /(\mathrm{Ac} / \mathrm{Pr})$ d'environ 10.

\section{6 / Influences combinées de l'apport de concentré et du niveau alimentaire}

Etant donné que ces deux facteurs sont connus pour être fréquemment en interaction en pratique, il a été décidé

Figure 8. Influence, calculée en intra-expérience, de la proportion d'aliment concentré sur le rapport acétate/propionate du jus de rumen.

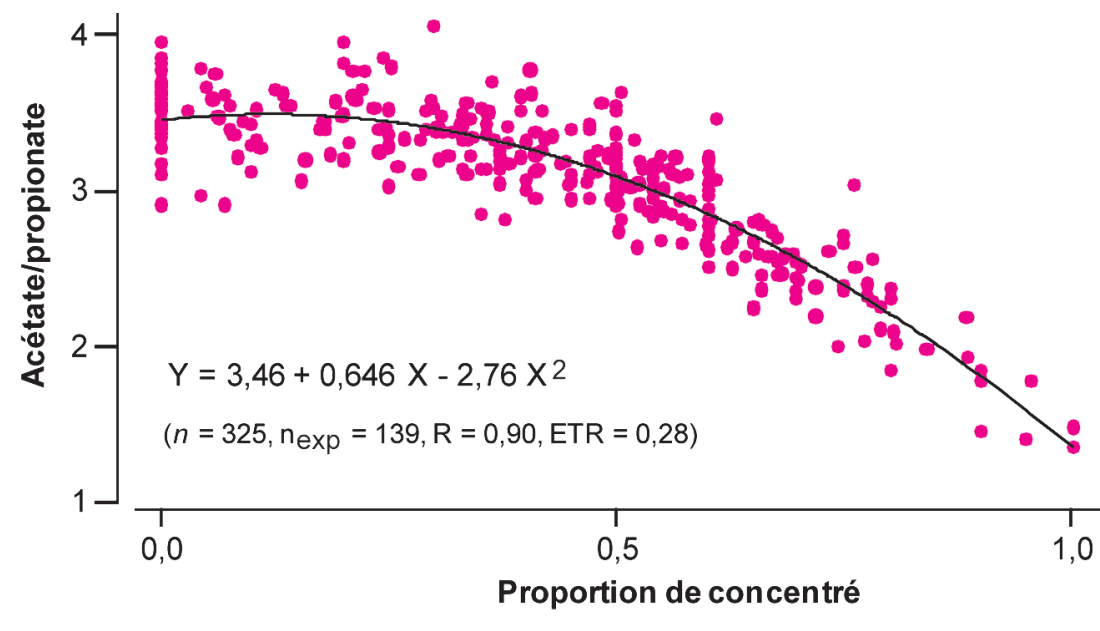


Figure 9. Influence, calculée en intra-expérience, de la proportion d'aliment concentré sur la production de $\mathrm{CH}_{4} / \mathrm{kg}$ de $\mathrm{MOD}$.

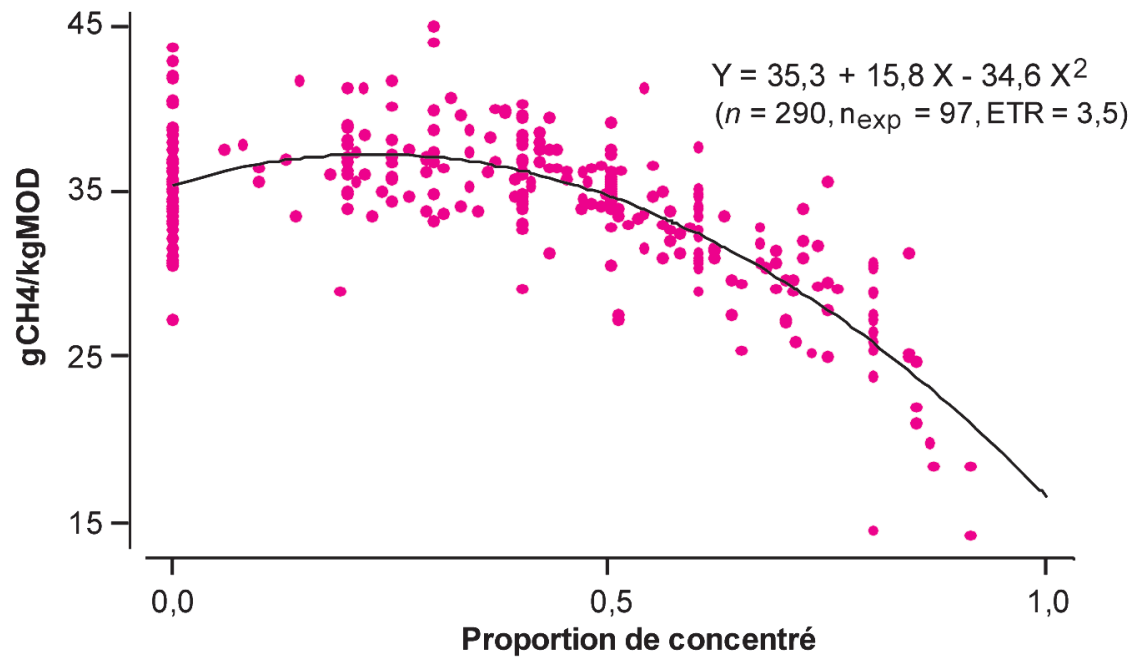

Base «Rumener» (UMR MoSAR), expériences portant sur l'influence du niveau de concentré

d'étudier leurs effets associés sur les fermentations. Au préalable, les métadispositifs liés à ces facteurs ont été étudiés de façon à s'assurer que les deux critères explicatifs étaient globalement indépendants, condition nécessaire pour pouvoir étudier valablement les effets d'interaction.

\section{a) Sur les profils fermentaires}

Les deux variables étudiées ont été la production de $\mathrm{CH}_{4}(26,3 \pm 5,5 \mathrm{~g} / \mathrm{kg}$ MOD, $\min =8,3$, Max $=36,2$ ) et la teneur en MOD $=663 \pm 75 \mathrm{~g} / \mathrm{kg}$ MSI, $\min =435$, Max $=889$ ). L'examen du méta-dispositif fourni par les données de la base «Bovidig» indique une indépendance satisfaisante entre les variations de NA, exprimées par MSI\%PV, et de PCO. Lorsque toutes les expériences de la base «Bovidig» consacrées à l'étusignificative entre ces deux facteurs, car le terme du produit est significatif.

$$
\begin{gathered}
\text { Ac } / \mathrm{Pr}=4,28-0,33 \mathrm{MSI} \% \mathrm{PV}+1, \mathbf{8 8} \mathrm{PCO} \\
-\mathbf{3 , 0 3} \mathrm{PCO}^{2}-\mathbf{0 , 2 7} \mathrm{MSI} \% \mathrm{PV} * \mathbf{P C O} \\
\left(n=422, \mathrm{n}_{\mathrm{exp}}=172, \mathrm{ETR}=0,29\right) \quad[8]
\end{gathered}
$$

La figure 10 indique la trace de cette régression avec un effet plus marqué du concentré lorsque le NA est élevé.

\section{b) Sur la production de $\mathrm{CH}_{4}$}

Lorsque toutes les expériences pordes interactions significatives sont également apparues entre les deux facteurs explicatifs et tous les termes quadratiques ont été significatifs : de du NA, ou de la PCO, sont prises en compte, il apparaît une interaction tant sur le PCO et le NA de la base «Rumener»ont été mises en commun,

Figure 10. Effets combinés du niveau alimentaire et de la proportion de concentré dans le régime sur les variations du rapport acétate/propionate dans le rumen.

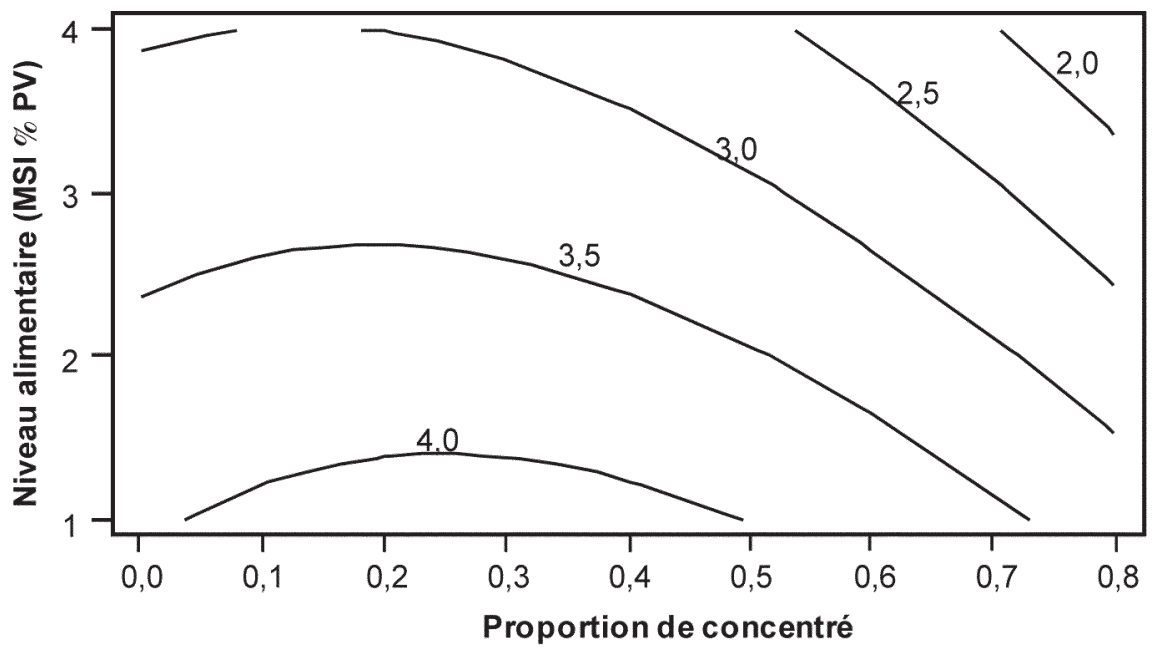

Base «Bovidig»

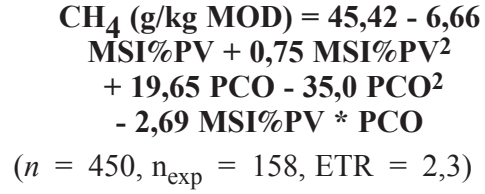

La figure 11 présente la trace de cette équation. Pour les niveaux alimentaires faibles, c'est-à-dire avec une ingestion de MS proche de $1 \%$ du PV, l'influence de l'apport de concentré sur $\mathrm{CH}_{4}$ en $\mathrm{g}$ par $\mathrm{kg}$ de MOD est peu marquée alors que, pour les niveaux alimentaires plus élevés, il apparaît une influence négative marquée.

Il existe une bonne cohérence entre les relations présentées sur les figures 10 et 11 , bien que les données ne soient pas issues des mêmes bases de données, confirmant ainsi indirectement la relation entre les profils fermentaires du rumen et la production de $\mathrm{CH}_{4}$. La simulation de valeurs de $\mathrm{Ac} / \mathrm{Pr}$ et de $\mathrm{CH}_{4}(\mathrm{~g} / \mathrm{kg} \mathrm{MOD}$ à partir de ces deux équations aboutit à un rapport $\mathrm{CH}_{4} / \mathrm{MOD} /[\mathrm{Ac} / \mathrm{Pr}]$ d'environ 10 , valeur comparable à ce qu'on observe avec les données de la base «Rumener» pour des valeurs de $\mathrm{Ac} / \mathrm{Pr}$ comprises entre 2 et 4 ( 55 traitements). La relation entre le profil en $\mathrm{AGV}$, évaluée par le rapport $\mathrm{Ac} / \mathrm{Pr}$, et la production de $\mathrm{CH}_{4} / \mathrm{MOD}$ présente donc une portée très générale et renforce la figure 2 . Ces résultats confirment bien que la production de $\mathrm{CH}_{4}$ dépend beaucoup des interactions digestives et ne peut donc pas être considérée comme un attribut des aliments (Sauvant et Giger-Reverdin 2009b), en particulier des aliments concentrés.

\section{7 / Influences de la nature du concentré : exemple de la vitesse de digestion de l'amidon}

Les principales variations de la digestion des ruminants en fonction de la nature et de la teneur en amidon des rations ont été décrites (Sauvant 1997). Peu de résultats publiés ont concerné cet aspect en relation avec la production de $\mathrm{CH}_{4}$. Les formes d'amidon rapidement dégradables sont digérées plus rapidement dans la panse et aboutissent à des $\mathrm{pH}$ plus faibles (Sauvant et Peyraud 2010) et des rapports Ac/Pr plus faibles donc une production probable moindre de $\mathrm{CH}_{4}$ par AGV. En revanche, compte tenu du niveau de fermentation nettement plus intense, une ration riche en amidon rapidement dégradable, orge par exemple, peut produire plus de $\mathrm{CH}_{4}$ qu'une forme d'amidon plus lentement dégradable, maïs grain par exemple (Beauchemin et McGinn 2005).

\section{8 / Influences de l'apport azoté}

La concentration du régime en Matières Azotées Totales (MAT) n'est 
Figure 11. Effets combinés du niveau alimentaire et de la proportion de concentré dans le régime sur la production de $\mathrm{CH}_{4}$ en $\mathrm{g} / \mathrm{kg} \mathrm{MOD.}$

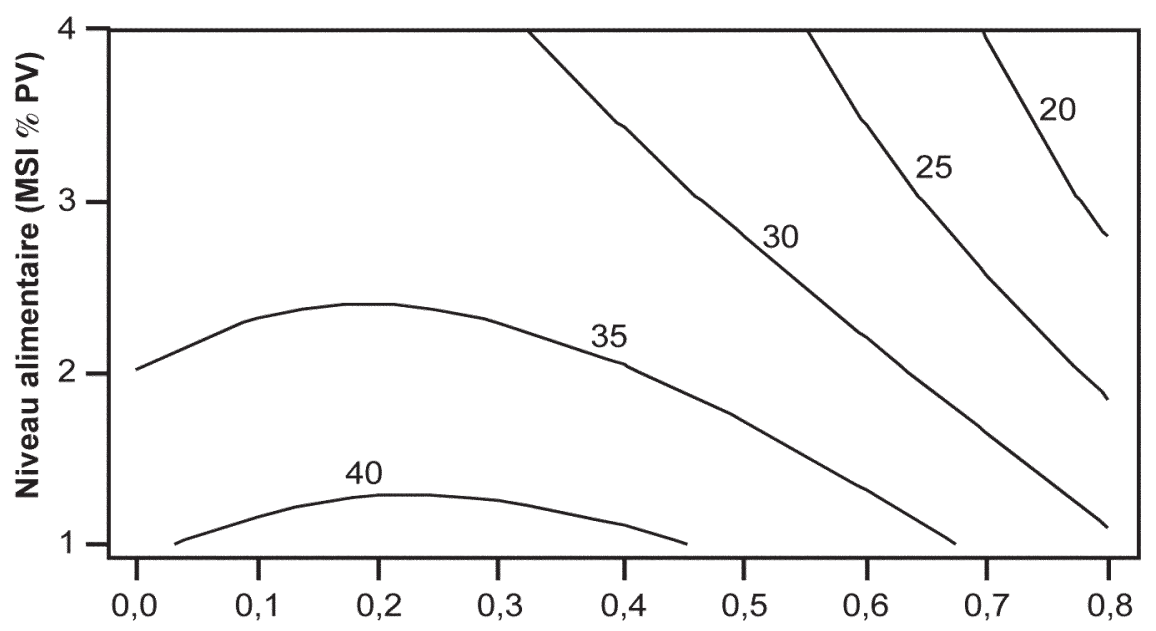

Proportion de concentré

pas réputée être un facteur de variation important de la production de $\mathrm{CH}_{4}$. Cependant, lorsque 59 expériences portant sur l'influence du taux de MAT sont sélectionnées dans la base «Rumener», il apparaît une influence négative significative du taux de MAT sur la production de $\mathrm{CH}_{4}$ (figure 12) :

$$
\begin{gathered}
\mathbf{C H}_{\mathbf{4}}(\mathrm{g} / \mathbf{k g} \text { MOD })=\mathbf{4 0 , 1}-\mathbf{0 , 3 2} \text { MAT\%MS } \\
\left(n=276, \mathrm{n}_{\mathrm{exp}}=59, \mathrm{ETR}=2,4\right)[10]
\end{gathered}
$$

Lorsque la production de $\mathrm{CH}_{4}$ est exprimée par rapport à la MSI, il n'y a pas de relation avec la teneur en MAT. Cette différence résulte du fait que l'accroissement de la teneur en MAT est associé à une amélioration significative de la teneur en MOD des rations considérées. En rervanche, sur la base «Bovidig», il n'apparaît pas de relation significative entre le rapport $\mathrm{Ac} / \mathrm{Pr}$ et la teneur en MAT du régime pour 56 expériences consacrées à cette question. Par ailleurs, avec la base «Rumener», il n'y a pas de relation entre le rapport $\mathrm{CH}_{4} / \mathrm{MOD}$ et la teneur en $\mathrm{N}-\mathrm{NH}_{3}$ du jus de rumen (18 expériences et 58 traitements). Enfin, sur cette même base et les mêmes essais sur l'influence du taux de MAT, il n'y a pas de relation intraexpérience entre les pertes d'énergie par le méthane $\left(\mathrm{ECH}_{4} \% \mathrm{~EB}\right)$ et par la voie urinaire $(\mathrm{EU} \% \mathrm{~EB})$ ce qui répond à certaines hypothèses faites en ce sens et facilite le calcul de la teneur en énergie métabolisable des rations de façon additive à partir de l'énergie digestible, des pertes d'énergie par le méthane et dans les urines, toutes ces grandeurs étant exprimées par rapport à l'énergie brute.

\section{9 / Influences de l'apport de lipides}

L'apport de Matières Grasses (MG) est souvent considéré comme l'un des moyens alimentaires les plus efficaces pour diminuer la production de méthane (Beauchemin et al 2007, Doreau et al 2011). Le plus souvent l'ingestion de MS est diminuée par une complémentation en lipides. De ce fait, les conclusions ne sont pas toujours les mêmes si l'on exprime les résultats par animal, par kg de MS ingérée ou par rapport à l'énergie digestible. Compte tenu du fait que les digestibilités ne sont pas toujours mesurées dans ces essais, les résultats sont exprimés par kg de MSI. Une première compilation de 7 publications sur vaches laitières avec 37 régimes avait mis en évidence, par régression globale, une diminution de la production de méthane avec l'augmentation de l'ingestion et du taux de lipides, mesuré par l'Extrait Ethéré (EE), deux variables indépendantes sur le jeu de données utilisé (Giger-Reverdin et al 2003) :

Figure 12. Influence, calculée en intra-expérience, de la teneur en MAT du régime sur la méthanogenèse.

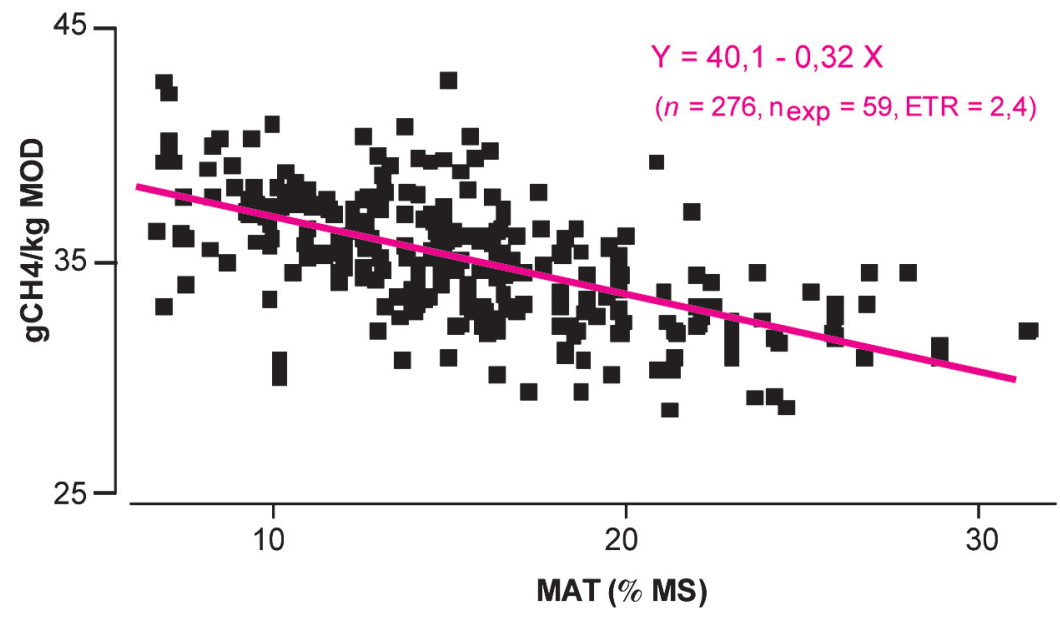

Base «Rumener» (UMR MoSAR) : relation «intra» d'expériences portant sur l'influence du taux de MAT la complémentation en lipides décroît le

$$
\begin{gathered}
\mathrm{CH}_{4}(\mathrm{~g} / \mathrm{kg} \mathrm{MS})=29,6-0,0133(\mathrm{MSI})^{2} \\
(\mathrm{~kg} \mathrm{MS})-0,00425 \mathrm{EE}(\mathrm{g} / \mathrm{kg} \mathrm{MS}) \\
\left(n=37, \mathrm{R}^{2}=0,76, \mathrm{ETR}=1,64\right)
\end{gathered}
$$

Cette diminution était essentiellement due à la fraction «AG» de la $\mathrm{MG}$ et était d'autant plus importante que la $\mathrm{MG}$ était insaturée, ce qui avait conduit ces auteurs à proposer d'inclure un index d'insaturation de la fraction lipidique. Cependant, bien que dans différentes expériences postérieures à cette étude, la production de méthane ait baissé avec l'augmentation de l'insaturation des lipides (Beauchemin et al 2007, Pilajun et al 2010), une compilation récente de 27 publications ne met pas en évidence cet effet (Grainger et Beauchemin 2011), mais donne un poids important à l'apport de lipides :

$$
\begin{gathered}
\mathrm{CH}_{4}(\mathrm{~g} / \mathrm{kg} \mathrm{MS})=24,65-0,103 \mathrm{~g} \mathrm{EE} \\
(\mathrm{g} / \mathbf{k g ~ M S}) \\
(n=92)
\end{gathered}
$$

Ces coefficients sont assez proches de ceux publiés par Moate et al (2011) qui s'appuient sur une base de données comprenant des publications communes :

$$
\begin{gathered}
\mathrm{CH}_{4}(\mathrm{~g} / \mathrm{kg} \mathrm{MS})=\mathbf{2 4 , 5 1 - 0 , 0 7 8 0 ~ E E} \\
(\mathrm{g} / \mathbf{k g ~ M S}) \\
\left(n=76, \mathrm{n}_{\exp }=17\right)
\end{gathered}
$$

Outre l'effet du niveau d'ingestion, l'apport de lipides peut modifier le fonctionnement ruminal, et en particulier, la digestion de la fraction glucidique, ce qui a des répercussions sur la production de $\mathrm{CH}_{4}$. Cet effet persisterait dans le temps (Grainger et Beauchemin 2011).

De façon cohérente avec ces résultats, rapport $\mathrm{Ac} / \mathrm{Pr}$ dans le rumen si bien 
qu'une relation significative apparaît entre la teneur en $\mathrm{AG}$ du régime $\mathrm{AG} \% \mathrm{MS}$ et le rapport Ac/Pr (Schmidely, communication personnelle) :

$$
\begin{gathered}
\mathbf{A c} / \mathbf{P r}=\mathbf{3 , 0}-\mathbf{0 , 0 6} \mathbf{A G} \% \mathbf{M S} \\
\left(n=85, \mathrm{n}_{\exp }=28, \text { ETR }=0,22\right)
\end{gathered}
$$

Les valeurs assez basses du rapport Ac/Pr proviennent du fait qu'il s'agit en général de données obtenues sur vaches laitières donc avec des régimes ingérés en grande quantité et comprenant des proportions importantes de concentrés. Donc l'action des lipides sur la méthanogenèse passe, en partie au moins, par un effet sur le profill des AGV dans le rumen. Il serait intéressant d'évaluer dans quelle mesure le rapport $\mathrm{Ac} / \mathrm{Pr}$ est sensible non seulement à la quantité mais également au degré d'insaturation des AG alimentaires.

\section{3 / Vers l'intégration de la méthanogenèse et des phé- nomènes digestifs au sein d'un modèle global de pré- vision}

\section{1 / Modèles mécanistes de la production de méthane}

Différents modèles ont été publiés pour pouvoir prévoir la production de $\mathrm{CH}_{4}$ par les ruminants. Ainsi, Benchaar et al (1998) ont proposé une prévision qui s'appuie à la fois sur des principes mécanistes et des régressions avec, en conclusion, un avantage à la modélisation mécaniste intégrant les principes de stœchiométrie présentés dans la partie 1 de ce texte. Plus récemment, Mills et al (2001) ont appliqué des principes comparables et ont proposé un modèle simple du métabolisme de $\mathrm{H}_{2}$ dans le rumen intégrable par exemple au sein d'un modèle mécaniste du rumen plus complet. Ce modèle applique des principes comparables à ceux des tableaux 1 et 2 avec des sources et des puits de $\mathrm{H}_{2}$, il inclut en plus une production et une utilisation d' $\mathrm{H}_{2}$ par les microbes selon qu'ils utilisent des acides aminés ou de l'azote non protéique pour leur croissance. Ces dernières hypothèses n'ont pas été étayées par des travaux récents et n'ont qu'un faible impact sur les bilans $\mathrm{H}_{2}$ calculés. La production de $\mathrm{CH}_{4}$ est calculée simplement par la différence entre le $\mathrm{H}_{2}$ produit et utilisé, donc le $\mathrm{H}_{2}$ disponible est impliqué à $100 \%$ dans la formation de $\mathrm{CH}_{4}$. Cette hypothèse sera discutée plus loin. Cette approche stœchiométrique présente l'inconvénient de dépendre de la qualité du modèle mécaniste de rumen «porteur». Or, les modèles mécanistes du rumen publiés jusqu'à présent sont peu précis (Offner et Sauvant 2006, Bannink et al 2008). En outre, Ellis et al (2010) ont bien montré les limites de ce modèle dans le cas de rations destinées à des bovins en croissance intensive.

\section{2 / Prévision de la production de méthane à partir des relations stoechiométriques et de la MO apparemment digérée dans le rumen}

Pour s'affranchir des limites de l'approche précédente qui dépendent de la qualité du modèle mécaniste de rumen utilisé, il convient de pouvoir s'appuyer sur plus de données expérimentales quantifiant la digestion dans le rumen. De nombreuses données publiées ont présenté des résultats de mesure des teneurs en MO apparemment digérée dans le rumen, ou MODRa = MOingérée - MOduodénale. Or, cette quantité de $\mathrm{MO}$ obtenue par différence entre la MO ingérée et la MO transitant au duodénum représente la $\mathrm{MO}$ fermentée dans le rumen en AGV et gaz, soit :

$$
\text { MODRa }=\text { MODR }_{A G V}+\text { MODRgaz }
$$

En conséquence, pour approfondir cette question, une sous-base de «Bovidig» regroupant 165 publications, 250 expériences et 643 traitements a été extraite avec, comme critères de sélection, les mesures de la MODRa et du profil molaire moyen des $\mathrm{AGV}$ du rumen. Les flux de MO ingérés et au duodénum ont été transformés en flux de $\mathrm{C}$ en supposant des teneurs moyennes en $\mathrm{C}$ des protéines et des glucides de 52 et $45 \%$ respectivement. Cette approche a été présentée par Nozière et al (2010) et Sauvant et al (2010).
Comme dans la plupart de ces expériences, la MO réellement fermentée (MODRr), équivalente de la MOF, avait été mesurée ; il est possible d'exprimer la production des AGV sur ce critère. Il apparait alors que la production moyenne d'AGV par kg de MODRr dans le rumen est de 8,01 $\pm 1,19$ moles/ gMODRr, valeur très proche des 8,03 moles obtenues par Nozière et al (2010) sur un jeu de données complètement différent, puisqu'il s'agissait de résultats de mesures effectuées avec des AGV marqués. Il convient de signaler que les variations inter-expériences du rapport AGV/MODRr sont étroitement et négativement liées à celles de l'efficacité de la croissance microbienne qui, on le sait, est entachée d'une certaine incertitude. Les productions des AGV calculées selon la démarche ci-dessus permettent de calculer, selon les coefficients stœchiométriques du tableau 2 , les productions de $\mathrm{CO}_{2}$, de $\mathrm{H}_{2}$, et de $\mathrm{CH}_{4}$ potentiel, en supposant que sa formation utilise tous les $\mathrm{H}_{2}$ formés. Ces valeurs de $\mathrm{CH}_{4}$ ont été rapportées à la teneur en MOD mesurée avec les mêmes régimes.

Dans une seconde phase, une confrontation a été effectuée entre ces prévisions de $\mathrm{CH}_{4}(\mathrm{~g} / \mathrm{kg}$ MOD potentiel et celles obtenues à partir des données mesurées avec la base «Rumener» en ne considérant que les expériences portant sur les influences de $\mathrm{PCO}$ et NA pour se placer au sein d'un même ensemble de pratiques alimentaires. Ces essais ont été ajustés dans «Rumener» pour produire l'équation [9] et celle-ci a été appliquée aux données de la base «Bovidig». La figure 13 montre qu'une relation intra-expérience curvilinéaire assez précise lie, en abscisse, le $\mathrm{CH}_{4}(\mathrm{~g} / \mathrm{kg}$ MOD potentiel, calculé avec les stœechiométries sur la base «Bovidig», avec en ordonnée, le

Figure 13. Relation entre la production de $\mathrm{CH}_{4}$ prédite par l'équation [9] et le calcul par stœchiométrie sur les mesures de MODRa et de profil d'AGV.

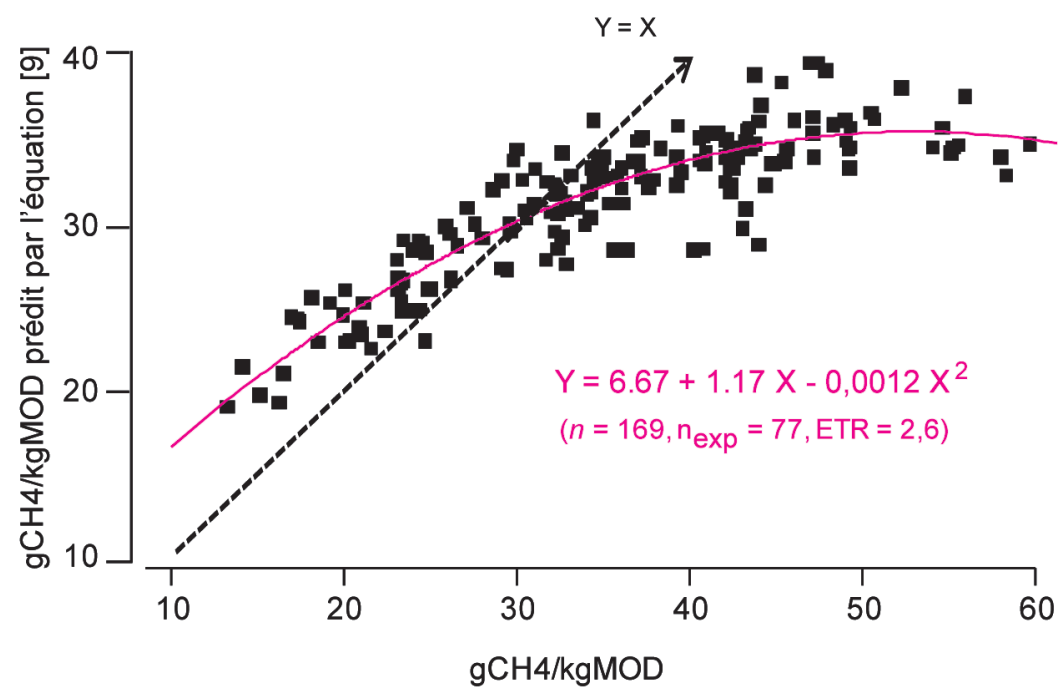

Base «Rumener» $(Y)$ et «Bovidig» $(X)$ 
$\mathrm{CH}_{4}(\mathrm{~g} / \mathrm{kg}$ MOD) prédit à l'aide de l'équation [9]. La position de la première bissectrice montre que les valeurs potentielles calculées sont égales aux valeurs prévues par l'équation [9] autour de la valeur de $30 \mathrm{gCH}_{4} / \mathrm{kg}$ MOD. Pour les valeurs inférieures, cas des rations les plus digestibles, riches en concentré, à efficacité microbienne et à rapport Ac/Pr faibles, les valeurs prévues par l'équation [9] sont plus élevées, alors que c'est l'inverse pour les valeurs supérieures à 30 , cas des régimes pauvres. Le fait que des valeurs calculées sont inférieures aux valeurs prévues peut provenir en partie du fait que la base «Rumener» ne concerne que des animaux placés en cage calorimétrique, où tout le $\mathrm{CH}_{4}$ produit est mesuré, alors que les valeurs calculées ne concernent que le rumen. Or, on sait que la production de $\mathrm{CH}_{4}$ par la partie distale du tube digestif du ruminant représente 8 à $16 \%$ du $\mathrm{CH}_{4}$ produit (Murray et al 1978). A ce stade, la question qui se pose est d'évaluer la part des facteurs interférents (MODRa/MOD et croissance microbienne) et des artéfacts de mesure dans ces variations. Au-delà de ce questionnement, la curvilinéarité de la relation a une portée générale, elle indique que le rendement marginal de la production calculée en production effective de $\mathrm{CH}_{4}$ serait de l'ordre de 1 pour les régimes riches en énergie et peu méthanogènes pour diminuer à mesure que les régimes fermentent moins et produisent à proportion plus de $\mathrm{CH}_{4}$. Cette curvilinéarité entre une production effective de $\mathrm{CH}_{4}$ et une production calculée à partir des principes de stœchiométrie a aussi été observée récemment in vitro (Serment et $a l$ non publié). Ce plafonnement de la production de $\mathrm{CH}_{4}$ pour les régimes méthanogènes, au-delà des principes de stœchiométrie, rappelle aussi la figure 2 . Ainsi trois approches indépendantes révèlent un plafonnement de la méthanogenèse, dans des conditions qui lui sont favorables, ce qui mérite étude. L'hypothèse d'une non-validité des relations stœchiométriques pour ces régimes peut être formulée. Elle pourrait s'expliquer par un puits $\mathrm{d}^{\prime} \mathrm{H}_{2}$ lié à des adaptations métaboliques des populations microbiennes : par exemple une acétogenèse croissante à mesure de l'excès d' $\mathrm{H}_{2}$ qui formerait de l'acétate sans $\mathrm{CH}_{4}$, l'évolution du profil des AGV allant dans ce sens. En conséquence, les approches qui supposent que tout l'hydrogène d'origine fermentaire est recyclé en $\mathrm{CH}_{4}$ (Mills et al 2001) semblent a priori surestimer d'autant plus la production de $\mathrm{CH}_{4}(\mathrm{~g} / \mathrm{kg} \mathrm{MOD})$ que le régime est pauvre en énergie.

\section{Conclusions}

Les résultats présentés montrent que la production de $\mathrm{CH}_{4}$ par l'écosystème du rumen est étroitement liée aux fermentations qui s'y déroulent. Elle résul- te des imbrications compliquées entre les métabolismes de l'énergie, de l'hydrogène et des bilans $\mathrm{C}$ des différentes voies fermentaires. Il apparaît ainsi que les microorganismes adaptent leurs activités fermentaires en fonction de l'équilibre entre la disponibilité en substrats fermentescibles et leurs besoins énergétiques. Dans ce contexte, la production de $\mathrm{CH}_{4}$, étroitement associée à celle des chaines d'AGV paires, constitue un moyen pour tirer le meilleur profit énergétique du substrat disponible. Cette adaptation, qui a dû prévaloir au cours de l'évolution, est compréhensible mais assez difficile à prévoir en pratique. De ce fait, elle est à l'origine d'une partie des difficultés rencontrées pour prévoir avec précision la production de $\mathrm{CH}_{4}$ par les ruminants.

De nombreuses équations globales de prévision de la production de $\mathrm{CH}_{4}$ ont été publiées dans la littérature. Elles traduisent l'influence des facteurs majeurs de variation de cette production. Cependant, pour les raisons évoquées, ces équations sont en général peu précises (Mills et al 2003). Beaucoup d'entre elles ont été calibrées, ou validées, sur un champ limité de données (d'un seul laboratoire par exemple), ce qui handicape les possibilités de généralisation, puisque les variations entre publications et/ou équipes sont très importantes en matière de production de $\mathrm{CH}_{4}$ D'autres équations mélangent plusieurs origines de données et types de facteurs (régimes variés, animaux divers...), mais ne corrigent pas en fonction de ces origines, ce qui aboutit à des modèles de prévision peu précis, car ils incluent les variations entre publications. Enfin, les modes d'expression de la production de $\mathrm{CH}_{4}$ rapportés à l'animal, ou à la MSI, ou à l'EB ignorent des variations liées aux différences de digestibilité entre les régimes et à l'adaptation de l'écosystème à la disponibilité en énergie.

Pour gagner en précision et en généricité dans ces modélisations empiriques, il paraît d'abord important de corriger au mieux les ajustements des effets publications, sachant qu'il importerait aussi de s'interroger sur le sens de cet effet publication traité en effet fixe. Si le but est d'appliquer un modèle robuste, "passe partout» et de précision satisfaisante, nous suggérons de tenir compte de la MOD ramenée à la MS équation [1] ou la MOD ingérée [1bis]. Pour plus de précision, il faut travailler au-delà de ces équations en se focalisant sur le critère $\mathrm{CH}_{4} / \mathrm{MOD}$ et en séparant les effets des facteurs représentatifs de différentes pratiques alimentaires. Ainsi, nous proposons des équations de prévision précises de la production de $\mathrm{CH}_{4}$ en fonction du niveau d'ingestion [4], des teneurs des régimes en concentré [7], en protéines brutes [10] et en lipides [11]. Ces équations sont établies sur de vastes ensembles de données et elles sont confortées par des équations cohérentes de prédiction du rapport Ac/Pr des AGV. En outre, dans le cas des fourrages, nous proposons une équation spécifique [5] permettant d'évaluer la production potentielle de $\mathrm{CH}_{4}$ à en attendre. Une fois que les principaux effets sont modélisés, et à condition que le méta-dispositif le permette, on peut chercher à les regrouper en mettant à jour leurs interactions. Une telle démarche a été proposée dans ce travail pour combiner les effets du niveau d'ingestion de MS et de la proportion de concentré [9].

A coté de ces équations empiriques qui ont montré leurs limites (Mills et al 2003), sont apparues des approches qui s'appuient plus sur les principes de stœchiométrie des fermentations décrits dans ce texte. Celles-ci sont a priori plus mécanistes et génériques, donc plus intéressantes. Cependant, la précision obtenue par ces approches dépend beaucoup de la qualité du modèle mécaniste qui prévoit les profils d'AGV, la proportion de MO fermentée dans le rumen par rapport à celle qui est digérée dans l'ensemble du tube digestif, et l'efficacité de la croissance microbienne. On sait que les modèles mécanistes publiés jusqu'à présent sont peu précis sur ces différents aspects et des progrès sont encore à réaliser sur la modélisation des relations entre les principaux paramètres de la digestion. En particulier, il conviendrait de pouvoir disposer de plus de résultats avec des mesures simultanées de ces trois critères majeurs. C'est pour éviter cet écueil des modèles mécanistes que nous avons cherché à travailler sur des données expérimentales de MODRa mesurées in vivo. Les résultats obtenus sont encourageants, cependant ils remettent en question l'hypothèse d'une utilisation à $100 \%$ du bilan d'hydrogène pour synthétiser $\mathrm{CH}_{4}$, en particulier pour les rations les plus méthanogènes.

Dans le cadre du projet «Systali» (projet d'amélioration des systèmes d'alimentation INRA pour les ruminants), les principales équations citées ci-dessus sont prises en compte dans la construction d'un modèle intégré du rumen qui permettra d'évaluer la production de $\mathrm{CH}_{4}$ en fonction des paramètres alimentaires classiques (niveau d'ingestion, proportion de concentré, teneur en matières azotées et en matières grasses...) et en fonction de paramètres intermédiaires (MOD, MOF...). Ce modèle présentera l'intérêt de prendre en compte au mieux les phénomènes d'interactions entre facteurs alimentaires et d'associer tous les autres paramètres de la digestion. 


\section{Références}

Alemu A.W., Dijkstra J., Bannink A., France J., Kebreab E., 2011. Rumen stoichiometric models and their contribution and challenges in predicting enteric methane production. Anim. Feed Sci. Technol., 166-167, 761-778.

Archimède H., Eugène M., Marie Magdeleine C., Boval M., Martin C., Morgavi D.P., Lecomte P., Doreau M., 2011 Comparison of methane production between $\mathrm{C} 3$ and $\mathrm{C} 4$ grasses and legumes. Anim. Feed Sci. Technol., 166-167, 59-64.

Bannink A., France J., Lopez S., Gerrits W.J.J., Kebreab E., Tamminga S., Dijkstra J., 2008. Modelling the implications of feeding strategy on rumen fermentation and functioning of the rumen wall. Anim. Feed Sci. Technol., 143, 3-26.

Baumont R., Dulphy J.P., Sauvant D., Tran G., Meschy F., Aufrère J., Peyraud J.L., Champciaux P., 2007. Les tables de la valeur des aliments. In : Alimentation des bovins, ovins et caprins. Besoins des animaux. Valeurs des aliments. Tables INRA 2007, Editions Quae, Versailles, France. 181-286.

Beauchemin K.A., McGinn S.M., 2005. Methane emissions from feedlot cattle fed barley or corn diets. J. Anim. Sci., 83, 653-661.

Beauchemin K.A., McGinn S.M., Petit H.V., 2007. Methane abatement strategies for cattle: Lipid supplementation of diets. Can. J. Anim. Sci., 87, 431-440.

Belaich J.P., 1986. Le rendement de la croissance et de la biomasse active dans les biotopes anaérobies. Reprod. Nutr. Develop., 26, 129145.

Benchaar C., Rivest J., Pomar C., Chiquette J., 1998. Prediction of methane production from dairy cows using existing mechanistic models and regression equations. J. Anim. Sci., $76,617-627$

Blümmel M., Aiple K.P., Steingass H., Becker K., 1999. A note on the stoichiometrical relationship of short chain fatty acid production and gas formation in vitro in feedstuffs of widely differing quality. J. Anim. Physiol. Anim. Nutr., 81, 157-167.

Chilliard Y., Martin C., Rouel J., Doreau M., 2009. Milk fatty acids in dairy cows fed whole crude linseed, extruded linseed, or linseed oil, and their relationship with methane output. J. Dairy Sci., 92, 5199-5211.

Czerkawski J.W., 1986. An introduction to rumen studies. Oxford, U.K., Pergamon Press, 236p.

Demeyer D.I., 1991. Quantitative aspects of microbial metabolism in the rumen and hindgut. In: Rumen microbial metabolism and ruminant digestion. Jouany J.P. (Ed). INRA Editions, Paris, France, 217-237.

Dollé J.B., Agabriel J., Peyraud J.L., Faverdin P., Manneville V., Raison C., Gac A., Le Gall A., 2011. Les gaz à effet de serre en élevage bovin : évaluation et leviers d'action. In : Gaz à effet de serre en élevage bovin : le méthane. Doreau M., Baumont R., Perez J.M. (Eds). Dossier, INRA Prod. Anim. 24, 415-432.
Doreau M., Martin C., Eugène M., Popova M., Morgavi D.P., 2011. Leviers d'action pour réduire la production de méthane entérique pa les ruminants. In : Gaz à effet de serre en élevage bovin : le méthane. Doreau M., Baumont R., Perez J.M. (Eds). Dossier, INRA Prod. Anim. 24, 461-474.

Ellis J.L., Dijkstra J., Kebreab E., Archibeque S., France J., Bannink A., 2010. Prediction of methane production in beef cattle within a mechanistic digestion model. In: $7^{\text {th }}$ Int. workshop on modelling nutrient digestion and utilisation in farm animals. Sauvant D., Van Milgen J., Faverdin P., Friggens N. (Eds) 10-12 September, Paris, France, 181-188.

Giger-Reverdin S., Morand-Fehr P., Tran G., 2003. Literature survey of the influence of dietary fat composition on methane production in dairy cattle. Livest. Prod. Sci., 82, 73-79.

Giger-Reverdin S., Sauvant D., Vermore M. Jouany J.P., 2000. Modélisation empirique des facteurs de variation des rejets de méthane par les ruminants. Renc. Rech. Rum., 7, 187190

Grainger C., Beauchemin K.A., 2011. Can enteric methane emissions from ruminants be lowered without lowering their production? Anim. Feed Sci. Technol., 166-167, 308-320.

INRA, 2007. Alimentation des bovins, ovins et caprins. Besoins des animaux. Valeurs des aliments, Tables INRA 2007. Editions Quae, Paris, France, 307p.

Janssen P.H., 2010. Influence of hydrogen on rumen methane formation and fermentation balances through microbial growth kinetics and fermentation thermodynamics. Anim. Feed Sci. Technol., 160, 1-22.

Meschy F., Bravo D., Sauvant D., 2004. Analyse quantitative des réponses des vaches laitières à l'apport de substances tampon. Inra Prod. Anim., 17, 11-18.

Mills J.A.N., Dijkstra J., Bannink A. Cammell S.B., Kebreab E., France J., 2001. A mechanistic model of whole-tract digestion and methanogenesis in the lactating dairy cow: model development, evaluation, and application. J. Anim. Sci., 79, 1584-1597.

Mills J.A.N., Kebreab E., Yates C.M., Crompton L.A., Cammell S.B., Dhanoa M.S. Agnew R.E., France J., 2003. Alternative approaches to predicting methane emissions from dairy cows. J. Anim. Sci., 81, 31413150 .

Moate P.J., Williams S.R.O., Grainger C. Hannah M.C., Ponnampalam E.N., Eckard R.J., 2011. Influence of cold-pressed canola, brewers grains and hominy meal as dietary supplements suitable for reducing enteric methane emissions from lactating dairy cows. Anim. Feed Sci. Technol., 166-167, 254-264.

Montoya J.C., Bhagwat A.M., Peiren N., De Campeneere S., De Baets B., Fievez V., 2011. Relationships between odd- and branchedchain fatty acid profiles in milk and calculated enteric methane proportion for lactating dairy cattle. Anim. Feed Sci. Technol., 166-167, 596602
Murphy M.R., Baldwin R.L., Koong L.J., 1982. Estimation of stoichiometric parameters for rumen fermentation of roughage and concentrate diets. J. Anim. Sci., 55, 411-421.

Murray R.M., Bryant A.M., Leng R.A., 1978. Methane production in the rumen and lower gut of sheep given lucerne chaff: effect of level of intake. Brit. J. Nutr., 39, 337-345.

Nozière P., Glasser F., Loncke C., Marty I.O., Vernet J., Sauvant D., 2010. Modelling rumen volatile fatty acids and its evaluation on net portal fluxes in ruminants. In: $7^{\text {th }}$ Int. workshop on modelling nutrient digestion and utilisation in farm animals. Sauvant D., Van Milgen J., Faverdin P., Friggens N. (Eds). 1012 September, Paris, France, 158-167.

Offner A., Sauvant D., 2006 Thermodynamic modeling of ruminal fermentations. Anim. Res., 55, 343-365.

Pilajun R., Wanapat M., Wachirapakorn C. Navanukroaw C., 2010. Effect of coconut oil and sunflower oil ratio on ruminal fermentation, rumen microorganisms, N-balance and digestibility in cattle. J. Anim. Vet. Adv., 9, 1868-1874

Popova M., Morgavi D.P., Doreau M., Martin C., 2011. Production de méthane et interactions microbiennes dans le rumen. In : Gaz à effet de serre en élevage bovin : le méthane Doreau M., Baumont R, Perez J.M. (Eds). Dossier, INRA Prod. Anim. 24, 447-460.

Sauvant D., 1997. Conséquences digestives et zootechniques de la digestion de l'amidon chez les ruminants. INRA Prod. Anim., 10, 287-300.

Sauvant D., Giger-Reverdin S., 2009a. Les variations du bilan carbone des ruminants d'élevage. Renc. Rech. Rum., 16, 229-232.

Sauvant D., Giger-Reverdin S., 2009b. Modélisation des interactions digestives et de la production de méthane chez les ruminants. INRA Prod. Anim., 22, 375-384.

Sauvant D., Peyraud J.L., 2010. Calculs de ration et évaluation du risque d'acidose. Inra Prod. Anim., 23, 333-342.

Sauvant D., Van Milgen J., 1995. Dynamic aspects of carbohydrate and protein breakdown and the associated microbial matter synthesis. In: Ruminant physiology: digestion, metabolism, growth and reproduction. Proc. $8^{\text {th }}$ Int Symp. Rum. Physiol., Von Engelhardt W. Leonhard-Marek S., Breves G. et Giesecke D. (Eds), Delmar Publishers, Albany, Germany, 71-91.

Sauvant D., Assoumaya C., Giger-Reverdin S., Archimède H., 2006. Etude comparative du mode d'expression du niveau d'alimentation chez les ruminants. Renc. Rech. Rum. 13, 103.

Sauvant, D., Schmidely, P., Daudin, J. J. StPierre N.R., 2008. Meta-analysis of experimental data in animal nutrition. Animal, 2, 1203-1214.

Wolin M.J., 1960. A theoretical rumen fermentation balance. J. Dairy Sci., 43, 14521459 


\title{
Résumé
}

Ce travail s'appuie sur l'étude de plusieurs bases de données en vue d'extraire des modèles de prévision de la production de $\mathrm{CH}_{4}$ en fonction des régimes et des fermentations ruminales. La méthanogenèse est décrite en relation avec les principaux principes de la stœchiométrie et de la thermodynamique des fermentations ruminales. Il apparaît en particulier une relation étroite entre la production de $\mathrm{CH}_{4}$ et le rapport des acides acétique/propionique du jus de rumen (Ac/Pr). Les variations du profil des AGV et de la production de $\mathrm{CH}_{4}$ traduisent des phénomènes d'adaptation des microorganismes du rumen à la quantité d'énergie disponible. Au sein des différents critères alimentaires de prévision de la production de $\mathrm{CH}_{4}$, la teneur en matière organique digestible (MOD) est intéressante : elle est globalement bien liée à la MO fermentescible du rumen, donc au $\mathrm{CH}_{4}$ produit, et à la valeur énergétique des aliments. Cependant, le rapport $\mathrm{CH}_{4} / \mathrm{MOD}$ varie également en fonction de certains facteurs de variation qui modifient aussi le rapport Ac/Pr dans le rumen : le niveau alimentaire, la qualité du fourrage, la teneur en concentré du régime et l'apport de matières grasses. Une dernière partie du texte est consacrée à l'étude de modèles plus mécanistes qui s'appuient sur les principes de stœechiométrie des AGV, ces modèles constituant une étape vers une modélisation intégrative de l'ensemble des phénomènes digestifs et fermentaires du rumen.

\begin{abstract}
Influences of diet and rumen fermentation on methane production by ruminants

This work is based on the study of several databases to extract predictive models of $\mathrm{CH}_{4}$ production based on diets and ruminal fermentations. Methanogenesis is described with the main principles of the stoichiometry and thermodynamics of ruminal fermentation. It appears a particularly positive relationship between $\mathrm{CH}_{4}$ production and the ratio of acetic / propionic in rumen liquor (Ac / Pr). Changes in the profile of the volatile fatty acids (VFA) and of $\mathrm{CH}_{4}$ production reflect phenomena of adaptation of the rumen microbes to the amount of available energy. Among the various criteria for predicting the food production of $\mathrm{CH}_{4}$, the digestible organic matter (DOM) is an interesting parameter to be considered: it is closely linked to the rumen fermentable $\mathrm{OM}$, therefore $\mathrm{CH}_{4}$, and the energy value of food. However, the ratio $\mathrm{CH}_{4} / \mathrm{DOM}$ also varies depending on dietary factors that also affect the ratio $\mathrm{Ac} / \mathrm{Pr}$ in the rumen: the feeding level, forage quality, the dietary concentrate and fat. A final section is devoted to the study of mechanistic models that are based on the principles of stoichiometry of VFA, these models constitute a step towards integrated modeling of all phenomena and digestive fermentation in the rumen..
\end{abstract}

SAUVANT D., GIGER-REVERDIN S., SERMENT A., BROUDISCOU L., 2011. Influences des régimes et de leur fermentation dans le rumen sur la production de méthane par les ruminants. In : Gaz à effet de serre en élevage bovin : le méthane. Doreau M., Baumont R., Perez J.M. (Eds). Dossier, INRA Prod. Anim., 24, 433-446. 